\title{
2dF-AAOmega spectroscopy of massive stars in the Magellanic Clouds
}

\section{The north-eastern region of the Large Magellanic Cloud ${ }^{\star}, \star \star$}

\author{
C. J. Evans ${ }^{1}$, J. Th. van Loon ${ }^{2}$, R. Hainich ${ }^{3}$, and M. Bailey ${ }^{4,2}$ \\ ${ }^{1}$ UK Astronomy Technology Centre, Royal Observatory, Blackford Hill, Edinburgh, EH9 3HJ, UK \\ e-mail: chris.evans@stfc.ac.uk \\ 2 Astrophysics Group, School of Physical and Geographical Sciences, Lennard-Jones Laboratories, Keele University, ST5 5BG, UK \\ 3 Institute for Physics and Astronomy, University of Potsdam, 14476 Potsdam, Germany \\ 4 Astrophysics Research Institute, Liverpool John Moores University, Liverpool Science Park ic2, 146 Brownlow Hill, \\ Liverpool L3 5RF, UK
}

Received 13 February 2015 / Accepted 5 August 2015

\begin{abstract}
We present spectral classifications from optical spectroscopy of 263 massive stars in the north-eastern region of the Large Magellanic Cloud. The observed two-degree field includes the massive 30 Doradus star-forming region, the environs of SN1987A, and a number of star-forming complexes to the south of 30 Dor. These are the first classifications for the majority (203) of the stars and include eleven double-lined spectroscopic binaries. The sample also includes the first examples of early OC-type spectra (AA $\Omega 30$ Dor 248 and 280), distinguished by the weakness of their nitrogen spectra and by C IV $\lambda 4658$ emission. We propose that these stars have relatively unprocessed CNO abundances compared to morphologically normal O-type stars, indicative of an earlier evolutionary phase. From analysis of observations obtained on two consecutive nights, we present radial-velocity estimates for 233 stars, finding one apparent single-lined binary and nine $(>3 \sigma)$ outliers compared to the systemic velocity; the latter objects could be runaway stars or large-amplitude binary systems and further spectroscopy is required to investigate their nature.
\end{abstract}

Key words. Magellanic Clouds - stars: early-type - stars: fundamental parameters - open clusters and associations: general

\section{Introduction}

Our knowledge of the massive-star populations of the Magellanic Clouds has increased significantly over the past decade, largely via observations with multi-object spectrographs (e.g. Massey \& Olsen 2003; Evans et al. 2004, 2006, 2011; Fariña et al. 2009; Lamb et al. 2013). Such surveys have been used to address questions pertaining to stellar evolution (e.g. Massey \& Olsen 2003; Evans et al. 2008), wide-area studies of stellar kinematics (e.g. Evans \& Howarth 2008), the formation of massive stars in relative isolation (e.g. Lamb et al. 2010; Bressert et al. 2012; Oey et al. 2013), the structure of stellar clusters (e.g. Hénault-Brunet et al. 2012a,b), the properties of the interstellar medium in the Clouds (e.g. Welty et al. 2006; van Loon et al. 2013), and, via multi-epoch observations, the binary properties of massive stars (e.g. Bosch et al. 2009; Sana et al. 2013; Dunstall et al. 2015).

The compendia of known spectral types of massive stars in the Clouds by Bonanos et al. (2009, 2010) emphasised the disparity in our knowledge of the spectral content of the Clouds, with classifications for 5324 stars in the Small Magellanic Cloud (SMC), but only 1750 in the Large Magellanic Cloud (LMC). Filtering the LMC catalogue from the Magellanic

\footnotetext{
* Tables 2-4 are available in electronic form at http://wWw . aanda.org

$\star \star$ Copies of the spectra are only available at the CDS via anonymous ftp to cdsarc.u-strasbg. fr (130.79.128.5) or via http://cdsarc.u-strasbg.fr/viz-bin/qcat?]/A+A/584/A5
}

Clouds Photometric Survey (MCPS, Zaritsky et al. 2004) by a faint magnitude limit of $V=15.3 \mathrm{mag}$ and a colour cut of $(V-I)<0.0$ mag to identify likely $\mathrm{O}$ - and luminous B-type objects gives a total of $\sim 10000$ stars $^{1}$. The true number of earlytype stars in the LMC will be influenced by the effects of crowding in the MCPS photometry and the number of interlopers included via the above filters, but there clearly remains much to learn about the massive-star content of the LMC, even in light of recent classifications for $780 \mathrm{O}$ - and B-type stars in 30 Doradus (Walborn et al. 2014; Evans et al. 2015).

In this article we present spectroscopy from observations in early 2006 (P.I. van Loon) to test the capabilities of the then new AAOmega spectrograph (Saunders et al. 2004; Sharp et al. 2006) on the $3.9 \mathrm{~m}$ Anglo-Australian Telescope (AAT). Two fields were observed in the LMC, one in the NE near the massive 30 Doradus star-forming region and a second in the NW, centred on the N11 region. Spectra for thirteen of the targets have been published to date: seven peculiar "nfp" stars (Walborn et al. 2010), a massive runaway O2-type star (Evans et al. 2010), and five eclipsing binary systems (Muraveva et al. 2014). Partly motivated by results from the VLT-FLAMES Tarantula Survey

\footnotetext{
1 In this illustrative calculation we adopt an absolute magnitude for a B0 dwarf of -3.6 (Walborn 1972), a modest extinction of $A_{V}=0.4 \mathrm{mag}$ (from a typical reddening toward the LMC of $E(B-V) \sim 0.13 \mathrm{mag}$ from Massey et al. 1995, and assuming $R$, the ratio of total-to-selective extinction of 3.1), and a distance modulus to the LMC of $18.5 \mathrm{mag}$ (Pietrzyński et al. 2013).
} 


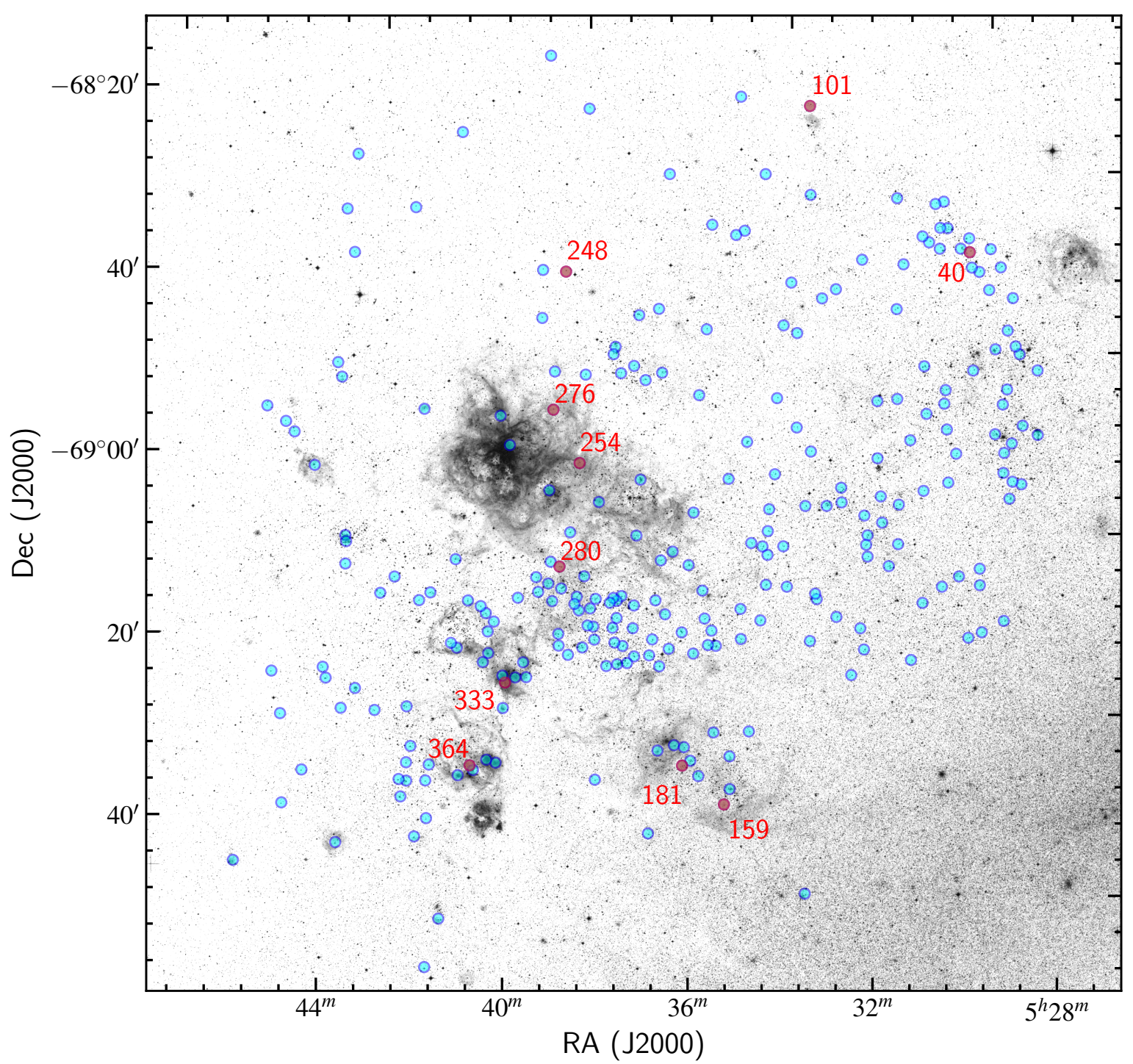

Fig. 1. Location of our AAOmega targets overlaid on a blue-optical image from the Digitized Sky Survey (DSS). The ten early-type stars (with classifications of $\mathrm{O} 4$ or earlier) discussed in Sect. 3 are highlighted in red.

(VFTS, Evans et al. 2011) regarding runaway stars (Evans et al. 2010, 2015), we have revisited the AAOmega data in the $\mathrm{NE}$ field to investigate the spectral content and dynamics of massive stars in this part of the LMC.

In this paper we present spectral classifications for 263 stars in the north-eastern region of the LMC, only 60 (23\%) of which have previous classifications (and often from observations of much lower quality and/or resolution). We describe the characteristics and reductions of the observations, including the optical photometry of our sources, in Sect. 2. The spectral classifications of our targets are discussed in Sect. 3, and estimates of stellar radial velocities (RVs) are presented in Sect. 4, followed by a short summary in Sect. 5. The observations in the field centred on the N11 region will be presented in a future article.

\section{Observations}

AAOmega is a fibre-fed, twin-arm spectrograph, with light separated into blue and red arms using a dichroic beam splitter (Saunders et al. 2004; Sharp et al. 2006). Up to 392 objects across a field on the sky that is $2^{\circ}$ in diameter can be observed simultaneously, using fibres on the prime-focus focal plate configured with the robotic positioner of the Two-degree Field facility (2dF, Lewis et al. 2002); each fibre has an onsky aperture of $2^{\prime \prime}$. Targets are selected in the $2^{\circ}$ field using the CONFIGURE software (see Lewis et al. 2002).

Luminous early-type stars were selected as potential targets from the MCPS (Zaritsky et al. 2004) using a magnitude cut of $V<14 \mathrm{mag}$ (to ensure a signal-to-noise ratio of $>50$ ) and a colour cut of $(V-I)<0.0$ mag to identify early-type stars. We used CONFIGURE to select targets from our input list using a field centre of $\alpha=05^{\mathrm{h}} 36^{\mathrm{m}} 07^{\mathrm{s}}, \delta=-69^{\circ} 15^{\prime} 58^{\prime \prime}(\mathrm{J} 2000)$. For context, these coordinates are $3^{\prime} \mathrm{NE}$ of the Honeycomb nebula (Wang 1992), 3'.5 E of SN1987A, and 17' SW of R136 (the massive cluster at the centre of 30 Dor $)$. The large field $\left(2^{\circ}\right.$ diameter $)$ enabled us to observe a reasonable number of targets in the regions immediately south of 30 Dor (including NGC 2060), in the N154, N158, and N160 complexes further to the south (Henize 1956), and field stars across the region. To illustrate the distribution of our targets, their locations are overlaid on the Digitized Sky Survey (blue-optical) image in Fig. 1.

The NE LMC field was observed on 2006 February 22-23. The data presented here were obtained with the blue arm, using the $1700 \mathrm{~B}$ grating on the first night, and the $1500 \mathrm{~V}$ grating on the 
Table 1. AAOmega spectrograph settings used.

\begin{tabular}{|c|c|c|c|c|c|}
\hline \multirow[t]{2}{*}{ Grating } & \multirow{2}{*}{$\begin{array}{c}\lambda_{\mathrm{c}} \\
{[\AA]}\end{array}$} & \multirow{2}{*}{$\begin{array}{c}\lambda \text {-coverage } \\
{[\AA]}\end{array}$} & \multicolumn{2}{|c|}{$\Delta \lambda(\mathrm{FWHM})$} & \multirow{2}{*}{$\begin{array}{c}\text { Exp. } \\
{[\mathrm{s}]}\end{array}$} \\
\hline & & & & [pix.] & \\
\hline & 410 & 376 & 1. & 3. & $2 \times 6$ \\
\hline & 470 & & 1.0 & 3. & \\
\hline $1500 \mathrm{~V}$ & 4375 & $3975-4755$ & 1.25 & 3.25 & $2 \times 900$ \\
\hline
\end{tabular}

second. Simultaneous observations were obtained with the red arm using the $1700 \mathrm{D}$ grating and centred at $8620 \AA$; this region contains fewer lines of interest for massive stars than the blue spectra so these data were not considered further.

The data were reduced using the 2DFDR software (Lewis et al. 2002). In brief, 2DFDR was used for bias subtraction, fibre location, extractions, division by a normalised flat-field, and wavelength calibration of each target. Subsequent processing included correction of the spectra to the heliocentric frame, sky subtraction, rejection of significant cosmic rays, and preliminary normalisation (using pre-defined continuum regions). The delivered spectral coverage and resolution from the observations with the blue arm of the spectrograph is summarised in Table 1. The signal-to-noise ratio of the final spectra obtained with the 1700B grating is 50-60 per rebinned pixel for the faintest targets, and in excess of 100 for the brightest supergiants. The signal-to-noise ratio of the (longer and slightly lower resolution) $1500 \mathrm{~V}$ observations is typically 20-30 greater than for the 1700B data.

Astrometry and optical photometry for each target (from the MCPS) is listed in Tables 2 and 3, respectively - the identifiers in the first column are simply the running numbers (in ascending RA) from our list of potential targets (hence those observed do not run in a continuous sequence); for consistency with the identifications used by Walborn et al. (2010) we also adopt them here. We note that the majority of the photometry from Zaritsky et al. for stars with $V<13.5$ mag was taken from the survey by Massey (2002), which employed a photometric aperture of 16.'2; thus in many instances crowding and/or nebular contamination may well influence the values in Table 3 (e.g. see discussion by Evans et al. 2011). Cross-matches of our targets with identifications/aliases from past spectroscopy are included in the final column of Table 2 .

\section{Spectral classification}

The AAOmega spectra were classified by visual inspection in comparison with standards following the usual precepts for early-type stars (Walborn \& Fitzpatrick 1990; Sota et al. 2011, 2014), taking into account the reduced metallicity of the LMC (e.g. Fitzpatrick 1988; Walborn et al. 1995, 2014; Evans et al. 2015), the effects of rotational broadening and the spectral resolution of our data. In brief, the primary diagnostic lines in the O-type spectra are the ionisation ratios of the helium lines, while also taking into account absorption from Si III at the latest types. The additional qualifiers employed in the classifications are summarised in Table 3 of Sota et al. (2011), and the spectral-type and luminosity-class criteria used for the later types are those summarised in Tables 4-6 of the same study. The B-type classifications employed the same spectral-type criteria as those in Tables 1 and 2 of Evans et al. (2015), with luminosity classes assigned from the width of the Balmer lines, while also taking into account the intensity of the silicon absorption lines at the earlier types; example sequences for $\mathrm{O}$ - and B-type spectra were given by Sota et al. (2011) and Evans et al. (2015), respectively.
The classifications of the AAOmega spectra are listed in Table 2, representing the first classifications for 203 of our targets. Previous classifications for the remaining 60 stars are summarised in Table 4 (complete to the best of our knowledge); in many cases the AAOmega spectra are superior to past spectroscopy, e.g. the resolution of the spectra obtained by Testor \& Niemela (1998) was only $8 \AA$ A. Initial inspection of the spectra revealed eleven double-lined binaries (SB2s), and a number of candidate single-lined binaries (SB1s), discussed further in Sect. 4.1.

Our classifications of the B-type supergiants include suffixes to indicate nitrogen lines which are strong (Nstr) or weak (Nwk) compared to morphologically-normal stars of the same adopted spectral type. These qualifiers were diagnosed from visual inspection of the CNO absorption features throughout the spectra, but principally informed by the intensity of the N II $\lambda 3995$ and the CNO features in the $\lambda \lambda 4640-4650$ region (Walborn 1976; Fitzpatrick 1991). An example of the contrast between Nstr and Nwk spectra of B-type supergiants is given in Fig. 1 of Evans et al. (2015).

The sample includes ten stars with classifications of $\mathrm{O} 4$ or earlier and their spectra are shown in Fig. 2. Four of these were previously unknown, namely: AA $\Omega 30$ Dor $101,181,248$, and 280. Given the absence of He I $\lambda 4471$ in the spectrum of $\mathrm{AA} \Omega 30$ Dor 159 , we adopt a classification of $03.5 \mathrm{III}\left(\mathrm{f}^{*}\right)$ over the O4 III(f) from Walborn et al. (2002a). The sample also includes examples of the nfp class of peculiar O-type spectra, which are defined by composite emission and absorption in the He II $\lambda 4686$ line (Walborn 1973). The six nfp stars in the sample, $\mathrm{AA} \Omega 30$ Dor 142, 187, 320, 333, 368 and 380, were included in the discussion of the phenomenon by Walborn et al. (2010), and we adopt their classifications here.

The classification of AA $\Omega 30$ Dor 078 (SOI 399, Stock et al. 1976) also merits brief discussion. Classified as A0 Ia by Stock et al., its classification here as B3 Iab is tantalysing in the context of variations associated with luminous blue variables. The objective-prism spectroscopy used by Stock et al. was relatively coarse in terms of spectral resolution, but it is notable that their classifications include spectra as early as B6 (for comparable magnitudes and from the same photographic plate), so it is plausible to assume that they would have been able to distinguish SOI 399 as a B-type spectrum if it was in the same state as the 2006 observations ${ }^{2}$. Checks of the ASAS-3 database (spanning 2000-2009, see Pojmański 2002) and the DASCH archive (spanning 1890-1990, Grindlay et al. 2012) reveal no significant photometric variations (given the cadence of the available data).

\subsection{Indications of CNO abundances in early O-type stars}

From inspection of the spectra in Fig. 2 we noted the apparent weakness of the nitrogen features in AA $\Omega 30$ Dor 248 and 280. These lines are the primary classification criteria at such early types (see Walborn et al. 2002a), yet in these two spectra there is only weak/marginal N III $\lambda \lambda 4634-40-42$ and N IV $\lambda 4058$ emission, and an absence of $\mathrm{N} \mathrm{V} \mathrm{\lambda \lambda 4604-4620} \mathrm{absorption.} \mathrm{The} \mathrm{ab-}$ sence/weakness of He I $\lambda 4471$ (and other He I features) argues for the early-types adopted here, and we suggest these are the nitrogen-poor counterparts of the morphologically normal and nitrogen-rich O2-type stars discussed by Walborn et al. (2004).

\footnotetext{
2 Stock et al. (1976) cross-matched their star 399 to $\mathrm{Sk}-68^{\circ} 100$, but Brian Skiff's updated catalogues (see footnotes to Table 2) match this Sanduleak source to SOI 398 (classified by Stock et al. as A1 Ia).
} 


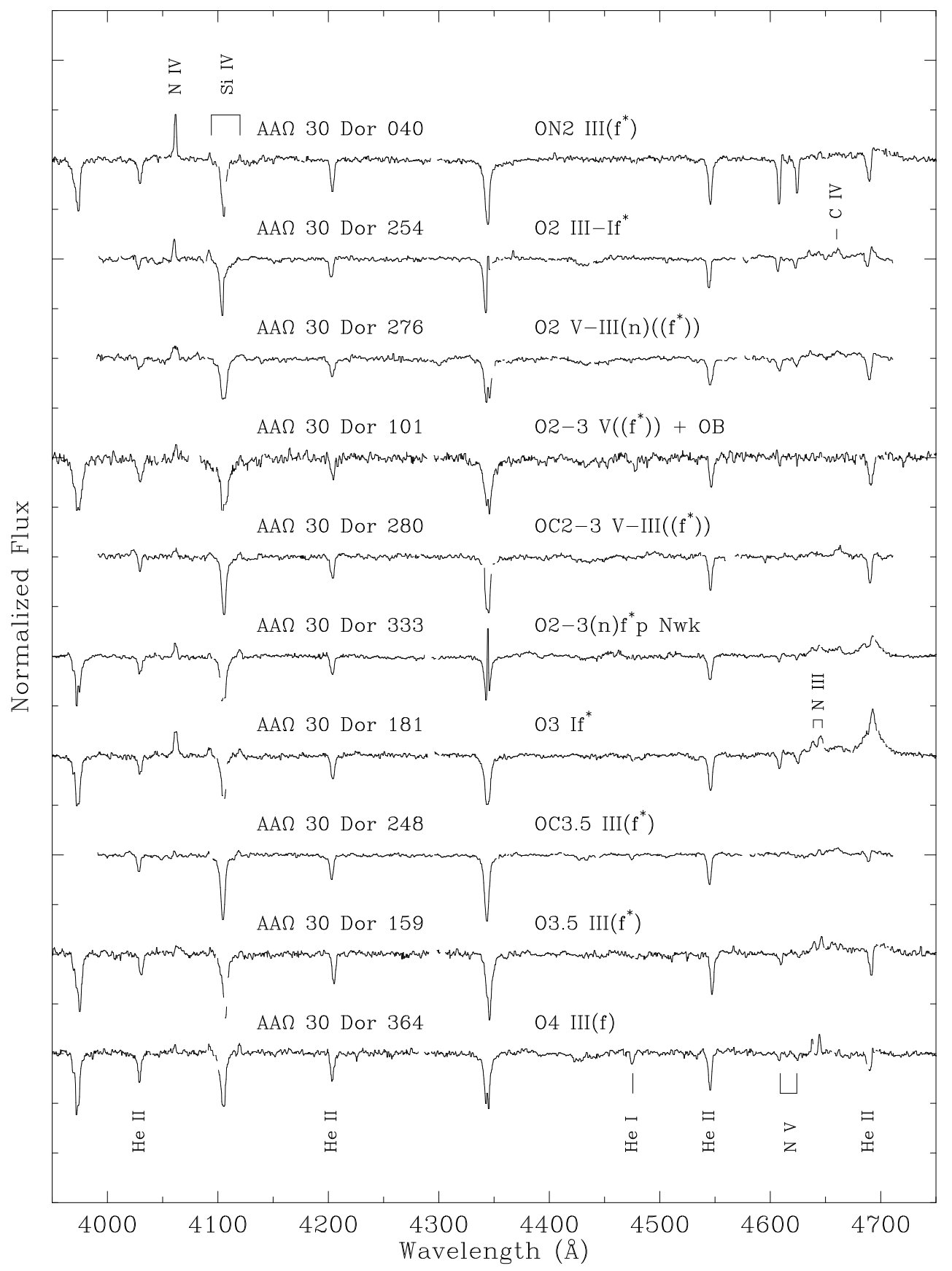

Fig. 2. AAOmega spectra of the ten early O-type stars in the sample (with each spectrum smoothed by a 5-pixel median filter for clarity and offset by 0.5 continuum units). Absorption lines identified in the spectrum of AA $\Omega 30$ Dor 364 are: He II $\lambda \lambda 4026,4200,4542,4686$; He I $\lambda 4471$; $\mathrm{N} v \lambda \lambda 4604,4620$. The emission lines identified in the spectra are, in order of increasing wavelength: N IV $\lambda 4058 ;$ Si IV $\lambda \lambda 4089,4116 ; \mathrm{N}$ III $\lambda \lambda 4634-$ 40-42; C IV $\lambda 4658$. Broad absorption from the $\lambda 4430$ diffuse interstellar band can be seen in some spectra (e.g. AA $\Omega 30$ Dor 254).

Nitrogen enrichment/deficiency in the spectra of late $\mathrm{O}$ - and early B-type spectra was first noted by Walborn (1976), primarily with reference to the absorption strengths of the $\mathrm{CNO}$ features in the $\lambda \lambda 4640-4650$ region. At earlier types, from both morphlogical considerations and quantitative analysis, Walborn et al. (2004) argued that some O2-type spectra (classified as ON2) are nitrogen-rich compared to morphologically normal O2-type spectra, suggesting a more advanced evolutionary state or greater chemical enrichment via initially-larger rotational velocities.

By analogy to the ON/OC sequence at later types (e.g. Walborn 1976; Sota et al. 2011), we therefore classify
AA $\Omega 30$ Dor 248 and 280 as OC-type spectra. As well as the chemical abundances, a broad range of physical factors (e.g. gravity and mass-loss rate) influence the appearance of the nitrogen features in the earliest O-type stars (Rivero González et al. 2012). On morphological grounds we employ the OC classification for the two spectra with an absence of $\mathrm{N} V \lambda \lambda 4604,4620$ absorption, combined with C IV $\lambda 4658$ emission. In addition, the weak but still discernable $\mathrm{N} \mathrm{V}$ absorption in the spectrum of AA $\Omega 30$ Dor 333 argues for an Nwk qualifier (Walborn, priv. comm.). As at later types, we suggest that AA $\Omega 30$ Dor 248 and 280 could be examples of an earlier evolutionary stage (i.e. less chemical processing/enrichment) than 
C. J. Evans et al.: AA $\Omega$ spectroscopy of massive stars in the NE of the LMC

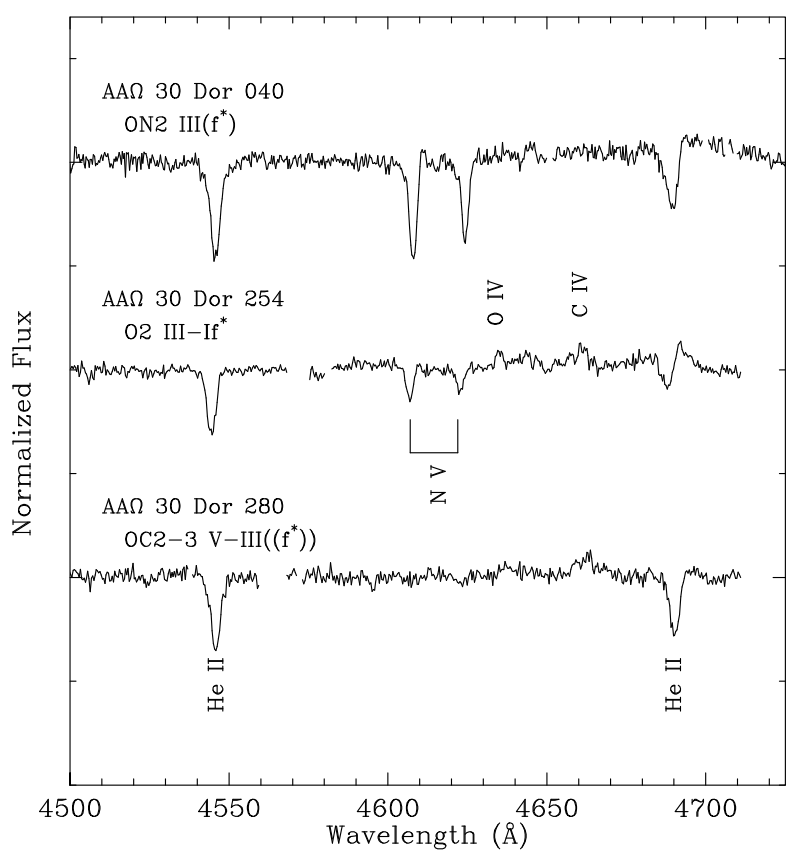

Fig. 3. $\lambda \lambda 4500-4725$ range of three early-type AAOmega spectra, illustrating the large variations in $\mathrm{N} v \lambda \lambda 4604-20$ absorption and the presence of C IV $\lambda 4658$ (and O IV $\lambda 4632$ ) emission.

the morphologically-normal objects such as AA $\Omega 30$ Dor 254, with the ON2 star, AA $\Omega 30$ Dor 040, completing the sequence. To highlight the trend in the $\mathrm{N} \mathrm{V}$ absorption lines in this sequence (and the presence of C IV emission), a subset region of the AAOmega spectra for three stars is shown in Fig. 3.

Pending further observations (including the $\mathrm{H} \alpha$ profiles to constrain the wind properties), we calculated investigative synthetic spectra using the PoWR model atmosphere code (Hamann 2003, 2004). Initially developed for analysis of WolfRayet type spectra, the code is also well suited for analysis of O-type stars (e.g. Oskinova et al. 2007; Evans et al. 2012). Adopting the physical properties from the published analysis of VFTS 016 (i.e. AA $\Omega 30$ Dor 254) from Evans et al. (2010), we calculated seven PoWR models to investigate if the C IV $\lambda 4658$ line is genuinely sensitive to abundance, or if it is significantly affected by other physical parameters.

The baseline model parameters were: an effective temperature $\left(T_{\text {eff }}\right)$ of $50 \mathrm{kK}$, luminosity $(L)$ of $\log \left(L / L_{\odot}\right)=6.08$, gravity $\left(g_{\text {grav }}\right)$ of $\log g_{\text {grav }}=3.75$, microturbulence $(\xi)$ of $30 \mathrm{~km} \mathrm{~s}^{-1}$, and a stellar wind with a terminal velocity $\left(v_{\infty}\right)$ of $3450 \mathrm{~km} \mathrm{~s}^{-1}$, an acceleration law described by a $\beta$ parameter of 1.0 , and with a mass-loss rate $(\dot{M})$ of $10^{-5.5} M_{\odot} \mathrm{yr}^{-1}$. Chemical abundances (by mass fraction) were $X_{\mathrm{H}}=0.7374, X_{\mathrm{He}}=0.258, X_{\mathrm{N}}=0.0008$, $X_{\mathrm{C}}=0.0008, X_{\mathrm{O}}=0.0016$. In the six panels of Fig. 4 we show the effects, from top to bottom, of reducing the mass-loss rate to $10^{-7} M_{\odot} \mathrm{yr}^{-1}$, reducing the terminal velocity to $1000 \mathrm{~km} \mathrm{~s}^{-1}$, introducing a clumped wind with a volume-filling factor, $f=0.1$ (i.e. $10 \%$ ), decreasing the microturblence to $15 \mathrm{~km} \mathrm{~s}^{-1}$, reducing the iron abundance by a factor of two, and reducing the carbon abundance by a factor of ten; changing $\beta$ by \pm 0.2 also had minimal impact. All of the spectra have been convolved with a rotational broadening profile of $150 \mathrm{~km} \mathrm{~s}^{-1}$, to match that adopted by Evans et al. (2010). These preliminary tests suggest that the carbon abundance is the principal factor influencing the appearance of the C IV line (for this adopted temperature and luminosity),

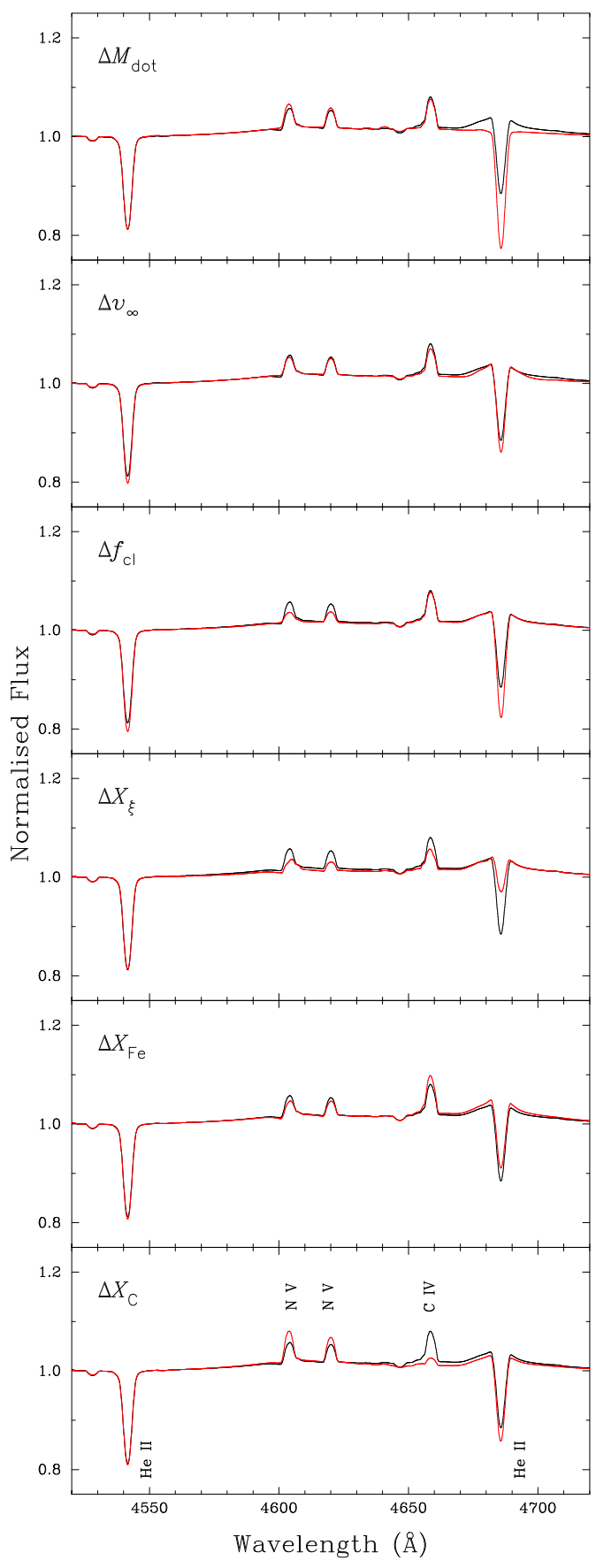

Fig. 4. Synthetic PoWR spectra (black line) adopting parameters for $\mathrm{AA} \Omega 30$ Dor 254 from Evans et al. (2010). The spectra plotted in red, moving from the top to bottom, are models which vary the mass-loss rate $\left(M_{\mathrm{dot}}\right)$, terminal velocity $\left(v_{\infty}\right)$ clumping factor $\left(f_{\mathrm{cl}}\right)$, microturbulence $(\xi)$, iron abundance ( $X_{\mathrm{Fe}}$, to investigate possible blanketing effects), and carbon abundance $\left(X_{\mathrm{C}}\right)$, as detailed in Sect. 3.1. The lines identified in the lower panel are He II $\lambda \lambda 4542,4686 ; \mathrm{N} v \lambda \lambda 4604-20$, and C IV $\lambda 4658$.

and that our hypothesis of these stars as carbon-rich (relative to nitrogen) is plausible ${ }^{3}$.

3 The PoWR models are not tailored to fit our spectra (e.g. in contrast to the observations, the $\mathrm{N} v$ lines are predicted in emission for the adopted parameters); our objective was a first investigation of the sensitivity of the C IV emission to different parameters. 


\section{Stellar radial velocities}

To estimate RVs for the O- and B-type stars we used Gaussian fits of the absorption lines listed in Table 5. The lines selected are those used by Sana et al. (2013) and Evans et al. (2015) to analyse the VFTS data, although we chose not to use He $\lambda 4026$ (blend of He I and II) nor He I $\lambda 4121$ (blend with O II). We note that He I $\lambda 4471$ was also avoided as it is typically the line most affected by nebular contamination, and also presents other discrepancies for RV estimates, probably due to its nature as a triplet transition and a possible blend with O II (for further discussion see Appendix B of Sana et al. 2013).

These adopted lines were not available at the earliest and latest types in the AAOmega sample. Thus, for the earliest O-type stars $(\leq \mathrm{O} 4)$ we used He II $\lambda \lambda 4026,4200,4542^{4}$. For the latertype (B9 and A-type) spectra we used $\mathrm{H} \delta$ and $\mathrm{H} \gamma$, combined with Si II $\lambda \lambda 4128-31$ and He I $\lambda 4471$.

Mean RVs for each target for the two nights, $v_{1}$ and $v_{2}$, are presented in Table 2. The quoted uncertainties are the standard errors (s.e.) on the means, and the sixth and eighth columns give the number of measured lines $(n)$; mean values are only calculated for spectra where $n \geq 3$. As a check for systematics in our measurements (e.g. line blends) and the adopted rest wavelengths, for each line in our analysis we calculated the mean residuals ( $\Delta_{\text {Night1 }}$ and $\Delta_{\text {Night2 }}$, and their standard deviations $)$ compared to the estimated RV for each star; these are given in the third and fourth columns of Table 5. In general, there are no significant systematic offsets present in the adopted lines. The Balmer lines in the cooler spectra appear to yield (marginally) different RV estimates, but these values are sufficient for our purposes.

\subsection{Binaries}

Our observations were obtained on two (consecutive) nights so our velocity estimates are somewhat limited in the search for variations arising from single-lined binaries. Nonetheless, we employed similar criteria to those used by Sana et al. (2013) and Dunstall et al. (2015) for identification of spectroscopic binaries from the VFTS. For stars with RV estimates from both nights, we consider it as a spectroscopic binary if they satisfy that $\left|v_{1}-v_{2}\right|>20 \mathrm{~km} \mathrm{~s}^{-1}$ between the observations and that $\left|v_{1}-v_{2}\right| / \sqrt{\sigma_{1}^{2}+\sigma_{2}^{2}}>4.0$, i.e. such variations are statistically significant, following the approach taken by Sana et al. (2013). The choice of the RV threshold for binary detection is a trade-off between the number of false positives arising from the effects of pulsations (and other atmospheric variations) and detections of real RV shifts from binary motion, as discussed by Sana et al. (2013) and Dunstall et al. (2015).

Employing these criteria, the RV estimates for only one star, $\mathrm{AA} \Omega 30$ Dor 053 (classified as B0.5 Ib Nwk), is formally significant as a SB1 system; this remains the case using a lower threshold of $\left|v_{1}-v_{2}\right|>16 \mathrm{~km} \mathrm{~s}^{-1}$ (as used by Dunstall et al. 2015, in their analysis of early B-type stars). There are a further six stars ${ }^{5}$ with $\left|v_{1}-v_{2}\right|>20 \mathrm{~km} \mathrm{~s}^{-1}$, but with sufficient uncertainties on their RV estimates that they do not satisfy the second criterion; for the purposes of the calculations in the next section we exclude these potential (though unconfirmed) RV variables.

In summary, eleven SB2 systems were found in our spectroscopy (with an additional SB2 candidate, $\mathrm{AA} \Omega 30$ Dor 173),

\footnotetext{
4 At such types $\lambda 4026$ is strongly dominated by He II absorption.

5 For completeness: AA $\Omega 30$ Dor 084, 085, 135, 160, 192, and 419.
}

Table 5. Rest wavelengths used for the radial-velocity estimates.

\begin{tabular}{lccc}
\hline \hline Ion & $\lambda$ & $\Delta_{\text {Night1 }}$ & $\begin{array}{c}\Delta_{\text {Night2 }} \\
{\left[\mathrm{km} \mathrm{s}^{-1}\right]}\end{array}$ \\
\hline \multicolumn{4}{c}{ O-type $(\leq$ O $)$ stars: } \\
He II & 4025.60 & $2.0 \pm 10.8$ & $-1.5 \pm 9.3$ \\
He II & 4199.83 & $-1.0 \pm 6.6$ & $3.2 \pm 6.7$ \\
He II & 4541.59 & $-1.0 \pm 12.2$ & $-1.7 \pm 4.7$ \\
\hline \multicolumn{4}{c}{ O-type $(>$ O4) stars: } \\
He II & 4199.83 & $-1.7 \pm 12.4$ & $-1.9 \pm 9.0$ \\
He I & 4387.93 & $0.4 \pm 9.8$ & $0.0 \pm 8.4$ \\
He II & 4541.59 & $1.8 \pm 10.4$ & $-0.2 \pm 7.9$ \\
He II & 4685.71 & $2.2 \pm 10.7$ & $2.0 \pm 10.0$ \\
He I & 4713.15 & $-1.6 \pm 9.3$ & $\ldots$ \\
He I & 4921.93 & $-1.2 \pm 7.4$ & $\ldots$ \\
\hline \multicolumn{4}{c}{$B$-type stars $($ excl. B9): } \\
He I & 4009.26 & $0.8 \pm 8.8$ & $1.8 \pm 9.8$ \\
He I & 4143.76 & $1.0 \pm 7.1$ & $1.1 \pm 5.7$ \\
He I & 4387.93 & $-2.1 \pm 5.4$ & $-2.6 \pm 4.9$ \\
He I & 4437.55 & $-0.1 \pm 8.6$ & $0.5 \pm 9.2$ \\
Si III & 4552.62 & $-1.2 \pm 6.1$ & $-1.1 \pm 5.5$ \\
Si III & 4567.84 & $-0.7 \pm 5.2$ & $2.1 \pm 7.7$ \\
Si III & 4574.76 & $-0.9 \pm 7.3$ & $-1.8 \pm 7.0$ \\
He I & 4713.15 & $2.4 \pm 5.4$ & $\ldots$ \\
He I & 4921.93 & $0.7 \pm 6.3$ & $\ldots$ \\
\hline \multicolumn{4}{c}{ B9/A-type stars: } \\
H & 4101.73 & $-3.6 \pm 5.2$ & $-2.4 \pm 1.8$ \\
Si II & 4128.07 & $3.8 \pm 11.0$ & $4.5 \pm 9.0$ \\
Si II & 4130.89 & $-0.3 \pm 3.2$ & $0.9 \pm 10.9$ \\
H & 4340.47 & $-3.7 \pm 3.3$ & $-4.2 \pm 7.6$ \\
He I & 4471.48 & $2.3 \pm 10.2$ & $0.3 \pm 5.0$ \\
\hline & \multicolumn{4}{c}{}
\end{tabular}

Notes. Values in the two final columns are the mean differences $(\Delta)$ between the velocity estimates from each line and the final velocity for each star. Wavelengths are from the NIST atomic spectra database (Kramida et al. 2012).

one SB1 system (AA $\Omega 30$ Dor 053), and six potential RV variables; these objects and their spectral classifications are listed in Table 6. Eight are known eclipsing systems from Graczyk et al. (2011) from the third phase of the Optical Gravitational Lensing Experiment (OGLE). Using a $2^{\prime \prime}$ search radius we then crossmatched our remaining targets with the Graczyk et al. (2011) catalogue, finding eight other eclipsing systems that were undetected as binaries from the available spectra. The OGLE identifiers, periods, and light-curve classifications are included in Table 6. Similar cross-checks with the luminous variables in the LMC reported by Szczygieł et al. (2010) yielded only one match - AA $\Omega 30$ Dor 286 (Sk-69 238), classified as O7.5 Ib(f), which was detected as a low-amplitude $(\sim 0.25 \mathrm{mag})$ photometric variable.

\subsection{Velocity distributions and radial-velocity outliers}

We calculated mean velocities of all our targets with RV estimates, and for subsamples limited to the O- and BA-type spectra. The resulting means and their associated standard deviations are summarised in Table 7, and the systemic value is in good agreement with results from the VFTS (Evans et al. 2015). In calculation of these results we have excluded stars flagged as potential RV variables in the previous section and those identified as eclipsing binaries. We also identified and (by iteration) excluded $\mathrm{RV}$ outliers for both nights, defined as stars with: $|v-\bar{v}|>3 \sigma$. 
Table 6. Summary of known binaries and candidate radial-velocity variables in the AAOmega sample.

\begin{tabular}{|c|c|c|c|c|c|}
\hline Star & Spectral classification & $\mathrm{AA} \Omega$ status & OGLE LMC-ECL & $P_{\mathrm{OGLE}}[\mathrm{d}]$ & Type $_{\text {OGLE }}$ \\
\hline 010 & O9.5: + early B & SB2 & & & \\
\hline 021 & B0.5: + early B & SB2 & 16629 & 2.923299 & $\mathrm{EC}$ \\
\hline 024 & B1.5 Ib Nwk & & 16646 & 26.225620 & ED \\
\hline 027 & B0.5: + early B & SB2 & 16675 & 3.748640 & ED \\
\hline 038 & B1-1.5 V-III & $\ldots$ & 16881 & 3.167171 & ED/VAR \\
\hline 053 & B0.5 Ib Nwk & SB1 & $\cdots$ & & $\cdots$ \\
\hline 058 & B1-1.5 V-III & $\ldots$ & 17198 & 3.290830 & ED \\
\hline 065 & O9.2-9.5 V & & 17334 & 1.362346 & ESD \\
\hline 084 & B1.5 Ib Nwk & RV var? & 17823 & 4.585031 & $\mathrm{EC}$ \\
\hline 085 & B0 V & RV var? & $\ldots$ & $\ldots$ & $\ldots$ \\
\hline 114 & B0.2 III: + early B & SB2 & & & \\
\hline 122 & O9.5 III + B0: & SB2 & 18794 & 5.946846 & ED \\
\hline 130 & B0.2: + early B & SB2 & $\ldots$ & $\ldots$ & $\cdots$ \\
\hline 135 & B1.5 III-II & RV var? & $\ldots$ & $\ldots$ & $\ldots$ \\
\hline 160 & B1.5 V-III & RV var? & & & \\
\hline 173 & B0.5 Ib Nwk? & SB2? & 19892 & 4.682925 & ED \\
\hline 178 & O6 V((f)) & $\ldots$ & 19996 & 1.079433 & ED \\
\hline 192 & O8.5 IIn & RV var? & & & \\
\hline 267 & O9.5: II + early B & SB2 & 20901 & 1.554470 & ED \\
\hline 330 & O9: V-III + O9.5: V-III & SB2 & 21568 & 3.225450 & ED \\
\hline 337 & O9: V-III + O9.7: V-III & SB2 & $\because$ & & \\
\hline 352 & Mid-O V & $\ldots$ & 21844 & 100.374558 & ED \\
\hline 371 & O9.5 II & .. & 22166 & 1.759227 & ED \\
\hline 374 & Early B + early B & SB2 & 22270 & 5.414011 & ED \\
\hline 381 & O9 III & & 22429 & 100.032930 & ED/VAR \\
\hline 414 & O8.5 Iabf (+ OB?) & SB2 & $\cdots$ & $\cdots$ & $\cdots$ \\
\hline 419 & B1-1.5 I & RV var? & $\ldots$ & $\ldots$ & $\ldots$ \\
\hline
\end{tabular}

Notes. Details of eclipsing binaries known from the OGLE survey are from Graczyk et al. (2011); classifications of light-curve variability in the final column are: $\mathrm{EC}=$ contact eclipsing binary; $\mathrm{ED}=$ detached eclipsing binary; $\mathrm{ESD}=$ semi-detached eclipsing binary; $\mathrm{ED} / \mathrm{VAR}=\mathrm{detached}$ with additional variability superimposed. Classifications of the spectra for AA $\Omega 30$ Dor 084 and 374 were given by Muraveva et al. (2014), identified in their Table A3 as 1m0020n19615 and lm0031122987, respectively; the more detailed classifications given here now supercede those.

Table 7. Mean radial velocities and dispersions for the AA $\Omega$ sample for both nights $\left(\overline{v_{1}}\right.$ and $\overline{v_{2}}$, respectively), excluding known and candidate binaries (see Sect. 4.1) and stars with outlying velocities (Table 8).

\begin{tabular}{lcrcr}
\hline \hline Sample & $\overline{v_{1}} \pm$ s.d. $\left[\mathrm{km} \mathrm{s}^{-1}\right]$ & \multicolumn{1}{c}{$n$} & $\overline{v_{2}} \pm$ s.d. $\left[\mathrm{km} \mathrm{s}^{-1}\right]$ & \multicolumn{1}{c}{$n$} \\
\hline All & $274.1 \pm 16.4$ & 199 & $274.3 \pm 16.5$ & 194 \\
O-type & $275.1 \pm 19.5$ & 68 & $275.3 \pm 18.6$ & 61 \\
BA-type & $273.6 \pm 14.6$ & 131 & $273.8 \pm 15.5$ & 133 \\
\hline
\end{tabular}

The RV estimates of the nine outliers are summarised in Table 8. Both AA $\Omega 30$ Dor 254 and 383 only have estimates (from three or more lines) for one night, but qualitative comparison of the spectra from the two nights reveals no obvious shifts; indeed, the former of these two objects is VFTS 016, identified as a runaway star by Evans et al. (2010, including analysis of these data).

For completeness, we note that two stars, AA $\Omega 30$ Dor 248 and 282, are (marginal) outliers in the estimates from one of the nights, suggesting either small RV variations or simply that we are at the limit of the available data (given that relatively few lines were available for RV estimates for these stars). Following the suggestion by Walborn et al. (2002a) that $\mathrm{Sk}-68^{\circ} 137$ and BI 253 (VFTS 072, AA $\Omega 30$ Dor 276) might be massive runaways, the case of AA $\Omega 30$ Dor 248 is similarly intriguing given its location ( $22^{\prime} \mathrm{NNW}$ of R136, see Fig. 1). Indeed, if its OC-type classification does indicate an early evolutionary phase it raises the question of whether it is an ejected runaway, or formed more locally (in relative isolation given the apparent lack of a nearby star-forming region).

AA $\Omega 30$ Dor 159 (W61 28-23) is the second largest outlier, and we note that Massey et al. (2005) reported a comparable RV ( $\sim 350 \mathrm{~km} \mathrm{~s}^{-1}$, see their Fig. 9) from observations in 1999 January. Whether this is a genuine runaway, or just chance observations of similar RVs for a large-amplitude binary system will require further spectroscopic monitoring. Indeed, additional spectroscopy of each object in Table 8 will be required to ascertain their true status.

\section{Summary}

We have presented spectral classifications from optical spectroscopy with 2dF-AAOmega for 263 massive stars in the NE region of the LMC, together with RV estimates for 233 stars in the sample. Ten stars have classifications of $\mathrm{O} 4$ or earlier, with two (AA $\Omega 30$ Dor 248 and 280) classified as OC-type given the nitrogen deficiency of their spectra combined with carbon emission; this is the first time such effects have been seen at such early types.

The spectra of 11 of our targets reveal them as SB2 systems, with a possible contribution from a secondary component seen in one other spectrum. From analysis of the RVs estimated from consecutive nights we identified one SB1 system and six candidate RV variables. Eight of these 19 objects are known eclipsing binaries from the OGLE survey (Graczyk et al. 2011). Eight of our other targets were also classified as eclipsing systems by Graczyk et al. (2011), but were undetected as binaries from our spectroscopy. 
Table 8. Stars with outlying radial velocities, which are candidate large-amplitude binaries or runaway stars.

\begin{tabular}{|c|c|c|c|c|c|c|c|}
\hline Star & Spectral type & $\begin{array}{l}v_{1} \pm \text { s.d. } \\
{\left[\mathrm{km} \mathrm{s}^{-1}\right]}\end{array}$ & $\begin{array}{l}\left(v_{1}-\overline{v_{1}}\right) \\
{\left[\mathrm{km} \mathrm{s}^{-1}\right]}\end{array}$ & $\left|v_{1}-\overline{v_{1}}\right| / \sigma$ & $\begin{array}{l}v_{2} \pm \text { s.d. } \\
{\left[\mathrm{km} \mathrm{s}^{-1}\right]}\end{array}$ & $\begin{array}{l}\left(v_{2}-\overline{v_{2}}\right) \\
{\left[\mathrm{km} \mathrm{s}^{-1}\right]}\end{array}$ & $\left|v_{2}-\overline{v_{2}}\right| / \sigma$ \\
\hline 097 & O9 IIIn & $209.0 \pm 15.3$ & $-65.1 \pm 22.4$ & 4.0 & $214.7 \pm 9.5$ & $-59.6 \pm 19.1$ & 3.6 \\
\hline 159 & $\mathrm{O} 3.5 \mathrm{III}\left(\mathrm{f}^{*}\right)$ & $370.1 \pm 5.7$ & $96.0 \pm 17.4$ & 5.8 & $377.8 \pm 7.2$ & $103.5 \pm 18.0$ & 6.3 \\
\hline 195 & B1 Ib Nwk & $328.3 \pm 12.2$ & $54.2 \pm 20.4$ & 3.3 & $329.9 \pm 4.3$ & $55.6 \pm 17.1$ & 3.4 \\
\hline 254 & O2 III-If* & & & & $190.3 \pm 1.6$ & $-84.0 \pm 16.6$ & 5.1 \\
\hline 383 & $\mathrm{O} 9.2 \mathrm{Ib}$ & $189.0 \pm 10.1$ & $-85.1 \pm 19.3$ & 5.2 & & & \\
\hline 401 & B0.5 Ia & $222.5 \pm 7.5$ & $-51.6 \pm 18.0$ & 3.1 & $216.1 \pm 7.1$ & $-58.2 \pm 18.0$ & 3.5 \\
\hline 424 & B0.7 Ib Nwk & $192.2 \pm 10.2$ & $-81.9 \pm 19.3$ & 5.0 & $199.9 \pm 7.1$ & $-74.4 \pm 18.0$ & 4.5 \\
\hline 426 & O6.5 III(f) & $213.6 \pm 14.5$ & $-60.5 \pm 21.9$ & 3.7 & $220.7 \pm 12.0$ & $-53.6 \pm 20.4$ & 3.2 \\
\hline 428 & $\mathrm{O} 7.5 \mathrm{~V}((\mathrm{f}))$ & $166.4 \pm 5.8$ & $-107.7 \pm 17.4$ & 6.6 & $163.7 \pm 8.1$ & $-110.6 \pm 18.4$ & 6.7 \\
\hline
\end{tabular}

Notes. The adopted mean velocities $\left(\overline{v_{1}}\right.$ and $\overline{v_{2}}$ ) are those from the first entry of Table 7 (which exclude the above stars and candidate binaries). AA $\Omega 30$ Dor $254=$ VFTS 016, the runaway star reported by Evans et al. (2010).

Using a $3 \sigma$ threshold compared to the systemic velocity (and excluding the known and candidate binaries), the estimated RVs were used to identify nine RV outliers (Table 8). These are likely to be large-amplitude binaries or runaway stars, and follow-up spectroscopy is required to clarify their nature.

Acknowledgements. We are grateful to Nolan Walborn for his careful reading of the draft manuscript, and for his thoughts regarding the classification of the early-type spectra. We also thank the referee for their constructive comments, and Russell Cannon and Gary Da Costa for their help with the observations.

\section{References}

Bonanos, A. Z., Massa, D. L., Sewilo, M., et al. 2009, AJ, 138, 1003 Bonanos, A. Z., Lennon, D. J., Köhlinger, F., et al. 2010, AJ, 140, 416 Bosch, G., Terlevich, E., \& Terlevich, R. 2009, AJ, 137, 3437 Bressert, E., Bastian, N., Evans, C. J., et al. 2012, A\&A, 542, A49 Brunet, J. P., Imbert, M., Martin, N., et al. 1975, A\&AS, 21, 109 Conti, P. S., Garmany, C. D., \& Massey, P. 1986, AJ, AJ, 92 Dunstall, P. R., Dufton, P. L., Sana, H., et al. 2015, A\&A, 580, A93 Evans, C. J., \& Howarth, I. D. 2008, MNRAS, 386, 826

Evans, C. J., Howarth, I. D., Irwin, M. J., Burnley, A. W., \& Harries, T. J. 2004, MNRAS, 353, 601

Evans, C. J., Lennon, D. J., Smartt, S. J., \& Trundle, C. 2006, A\&A, 456, 623 Evans, C., Hunter, I., Smartt, S., et al. 2008, The Messenger, 131, 25 Evans, C. J., Walborn, N. R., Crowther, P. A., et al. 2010, ApJ, 715, L74 Evans, C. J., Taylor, W. D., Hénault-Brunet, V., et al. 2011, A\&A, 530, A108 Evans, C. J., Hainich, R., Oskinova, L. M., et al. 2012, ApJ, 753, 173 Evans, C. J., Kennedy, M., Dufton, P. L., et al. 2015, A\&A, 574, A13 Fariña, C., Bosch, G. L., Morrell, N., Barbá, R., \& Walborn, N. R. 2009, AJ, 138, 510

Fitzpatrick, E. L. 1988, ApJ, 335, 703

Fitzpatrick, E. L. 1991, PASP, 103, 1123

Graczyk, D., Soszyński, I., Poleski, R., et al. 2011, Acta Astron., 61, 103

Grindlay, J., Tang, S., Los, E., \& Servillat, M. 2012, in Opening the 100Year Window for Time-Domain Astronomy, eds. E. Griffin, R. Hanisch, \& R. Seaman, IAU Symp., 285, 29

Hamann, W.-R., \& Gräfener, G. 2003, A\&A, 410, 990

Hamann, W.-R., \& Gräfener, G. 2004, A\&A, 427, 697

Hénault-Brunet, V., Evans, C. J., Sana, H., et al. 2012a, A\&A, 546, A73

Hénault-Brunet, V., Gieles, M., Evans, C. J., et al. 2012b, A\&A, 545, L1 Henize, K. 1956, ApJS, 2, 315

Jaxon, E. G., Guerrero, M. A., Howk, J. C., et al. 2001, PASP, 113, 1130

Kramida, A., Ralchenko, Y., \& Reader, J. \& NIST ASD Team. 2012, in NIST Atomic Spectra Database (v5.0); http:/physics.nist.gov/asd (Gaithersburg, MD.: National Institute of Standards and Technology)

Lamb, J. B., Oey, M. S., Werk, J. K., \& Ingleby, L. D. 2010, ApJ, 725, 1886

Lamb, J. B., Oey, M. S., Graus, A. S., Adams, F. C., \& Segura-Cox, D. M. 2013, ApJ, 763, 101

Lewis, I. J., Cannon, R. D., Taylor, K., et al. 2002, MNRAS, 333, 279
Lucke, P. B., \& Hodge, P. W. 1970, AJ, 75, 171

Massey, P. 2002, ApJS, 141, 81

Massey, P., \& Olsen, K. A. G. 2003, AJ, 126, 2867

Massey, P., Lang, C. C., DeGioia-Eastwood, K., \& Garmany, C. 1995, ApJ, 438, 188

Massey, P., Waterhouse, E., \& DeGioia-Eastwood, K. 2000, AJ, 119, 2214

Massey, P., Puls, J., Pauldrach, A. W. A., et al. 2005, ApJ, 627, 477

Massey, P., Morrell, N. I., Neugent, K. F., et al. 2012, ApJ, 748, 96

Melnick, J. 1985, A\&A, 153, 235

Muraveva, T., Clementini, G., Maceroni, C., et al. 2014, MNRAS, 443, 432

Oey, M. S. 1996a, ApJS, 104, 71

Oey, M. S. 1996b, ApJS, 465, 231

Oey, M. S., Lamb, J. B., Kushner, C. T., Pellegrini, E. W., \& Graus, A. S. 2013, ApJ, 768, 66

Oskinova, O., Hamann, W.-R., \& Feldmeier, A. 2007, A\&A, 476, 1331

Parker, J. W. 1993, AJ, 106, 560

Pietrzyński, G., Graczyk, D., Gieren, W., et al. 2013, Nature, 495, 76

Pojmański, G. 2002, Acta Astron., 52, 397

Rivero González, J. G., Puls, J., Massey, P., \& Najarro, F. 2012, A\&A, 543, A95

Sana, H., de Koter, A., de Mink, S. E., et al. 2013, A\&A, 550, A107

Sanduleak, N. 1969, Contrib. Cerro Tololo Inter-American Obs., No. 89

Saunders, W., Bridges, T., Gillingham, P., et al. 2004, in Ground-based Instrumentation for Astronomy, eds. A. Moorwood, \& M. Iye, Proc. SPIE, 5492, 389

Schild, H., \& Testor, G. 1992, A\&AS, 92, 729

Sharp, R., Saunders, W., Smith, G., et al. 2006, in Ground-based and Airborne Instrumentation for Astronomy, eds. I. McLean, \& M. Iye, (Proc. SPIE), 6269, 62690G

Sota, A., Maíz Apellániz, J., Walborn, N. R., et al. 2011, ApJS, 193, 24

Sota, A., Maíz Apellániz, J., Morrell, N. I., et al. 2014, ApJS, 211, 10

Stock, J., Osborn, W., \& Ibañez, M. 1976, A\&AS, 24, 35

Szczygieł, D. M., Stanek, K. Z., Bonanos, A. Z., et al. 2010, AJ, 140, 14

Testor, G., \& Niemela, V. 1998, A\&AS, 130, 527

van Loon, J. T., Bailey, M., Tatton, B. L., et al. 2013, A\&A, 550, A108

Walborn, N. R. 1972, AJ, 77, 312

Walborn, N. R. 1973, AJ, 78, 1067

Walborn, N. R. 1976, ApJ, 205, 419

Walborn, N. R., \& Blades, J. C. 1997, ApJS, 112, 457

Walborn, N. R., \& Fitzpatrick, E. L. 1990, PASP, 102, 379

Walborn, N. R., Lennon, D. J., Haser, S., Kudritzki, R. P., \& Voels, S. A. 1995, PASP, 107, 104

Walborn, N. R., Howarth, I. D., Lennon, D. J., et al. 2002a, AJ, 123, 2754

Walborn, N. R., Maíz Apellániz, J., \& Barbá, R. 2002b, AJ, 124, 1601

Walborn, N. R., Morrell, N. I., Howarth, I. D., et al. 2004, ApJ, 608, 1028

Walborn, N. R., Howarth, I. D., Evans, C. J., et al. 2010, AJ, 139, 1283

Walborn, N. R., Sana, H., Simón-Díaz, S., et al. 2014, A\&A, 564, A40

Wang, L. 1992, The Messenger, 69, 34

Welty, D. E., Federman, S. R., Gredel, R., Thorburn, J. A., \& Lambert, D. L. 2006, ApJS, 165, 138

Westerlund, B. E. 1961, Uppsala Astron. Obs. Ann., 5, 1

Zaritsky, D., Harris, J., Thompson, I. B., \& Grebel, E. K. 2004, AJ, 128, 1606

Pages 9 to 19 are available in the electronic edition of the journal at http://www . aanda. org 
C. J. Evans et al.: AA $\Omega$ spectroscopy of massive stars in the NE of the LMC

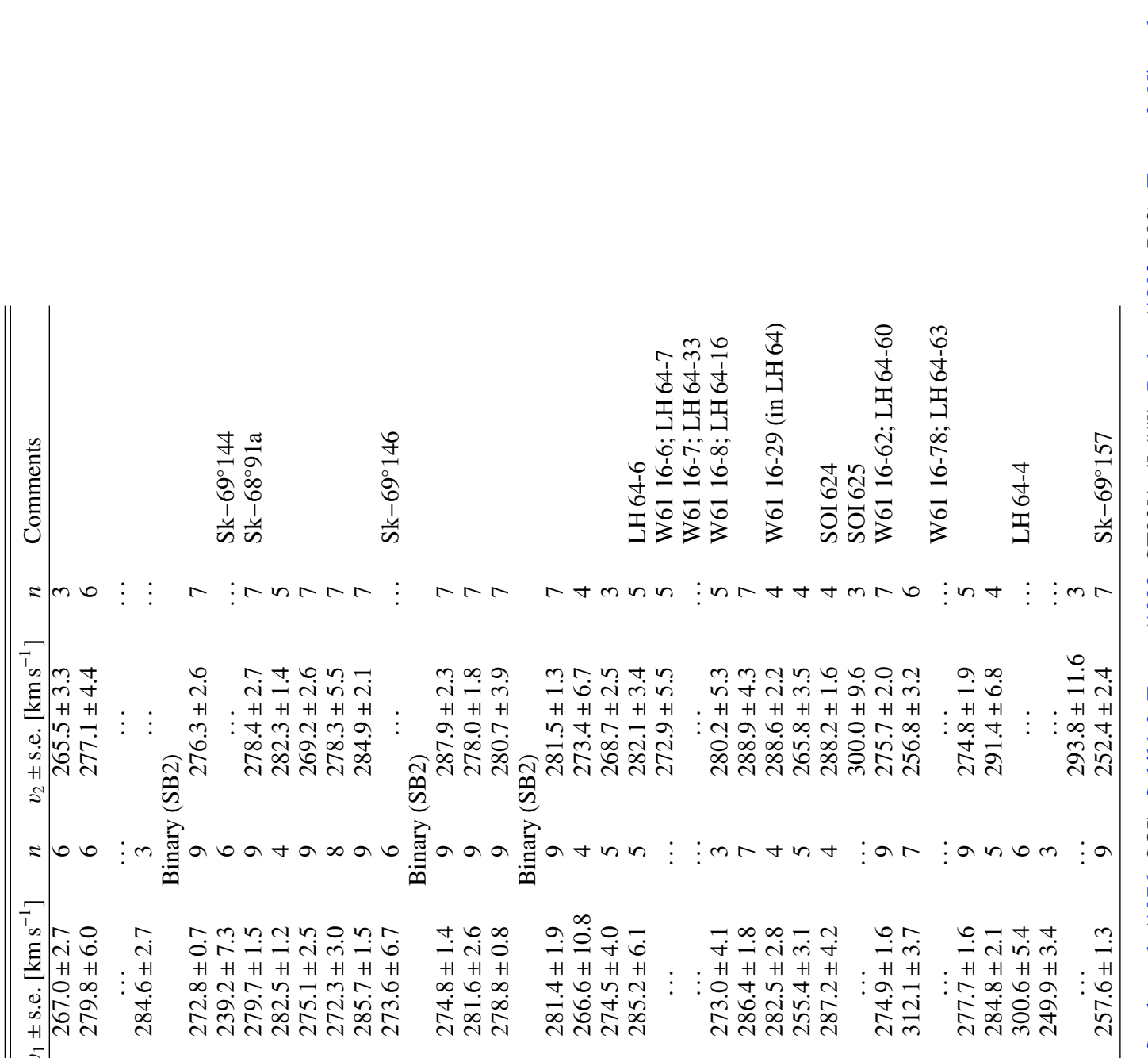

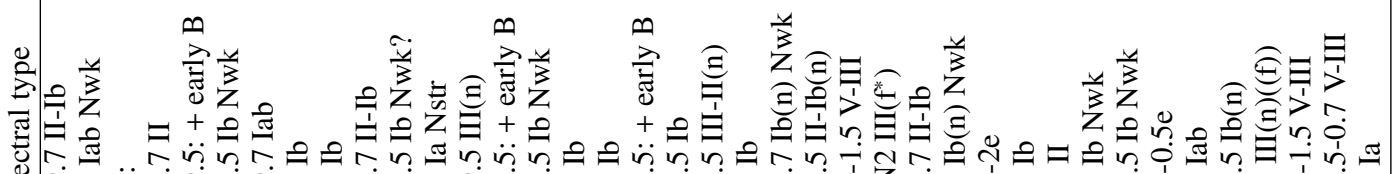

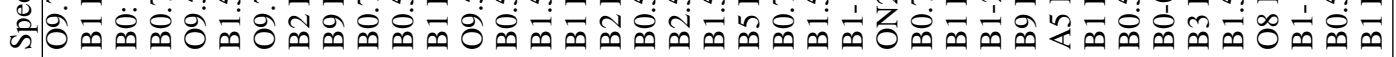

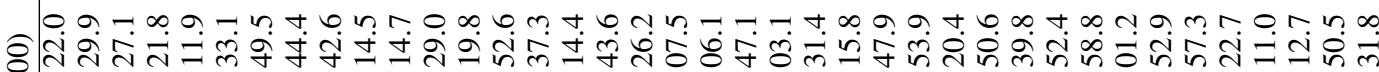

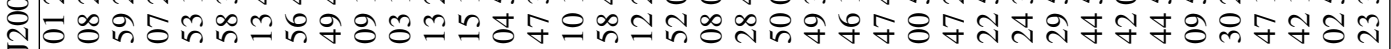

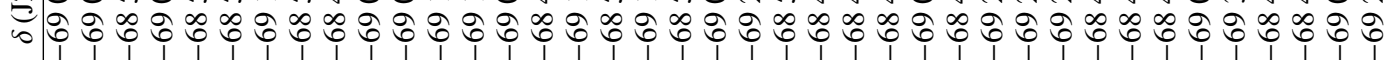

ชิ

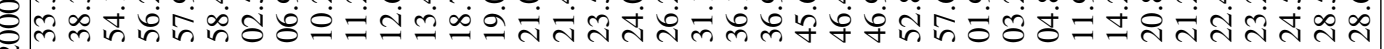

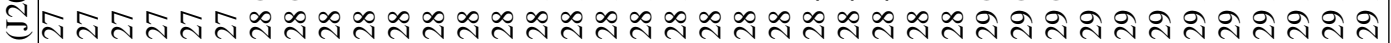

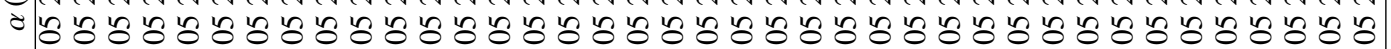

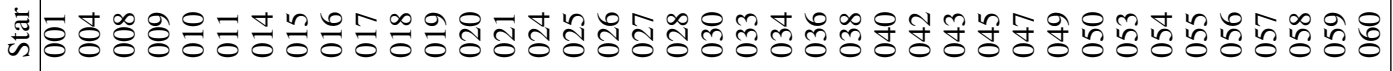

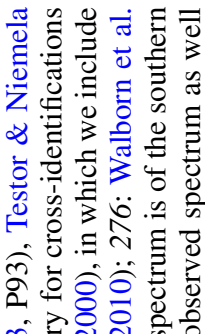

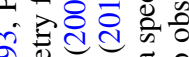

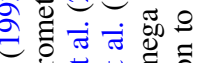

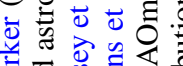

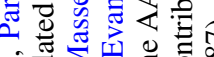

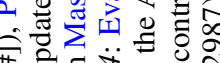

क

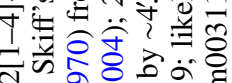

б. ज़

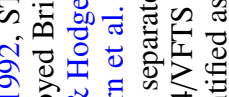

它义

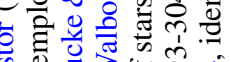

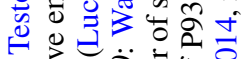

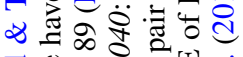

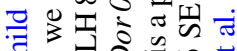

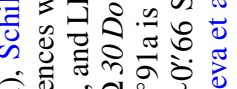

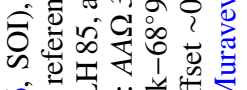

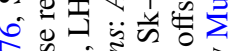

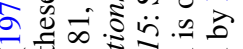

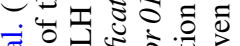
ए)

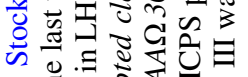

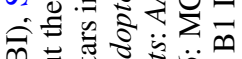
向 ผ气

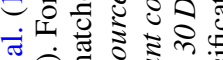

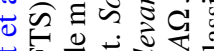

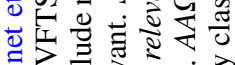

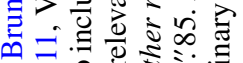

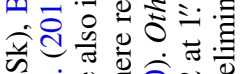
की

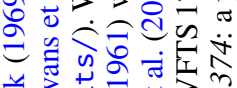

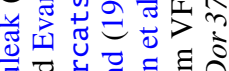

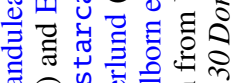
जิ

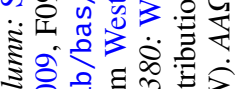

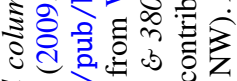

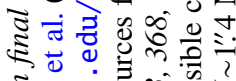
इ

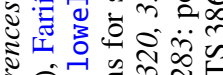

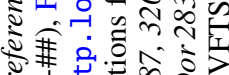

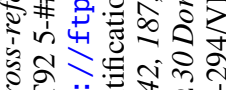

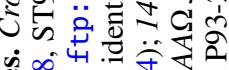

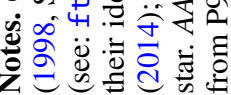




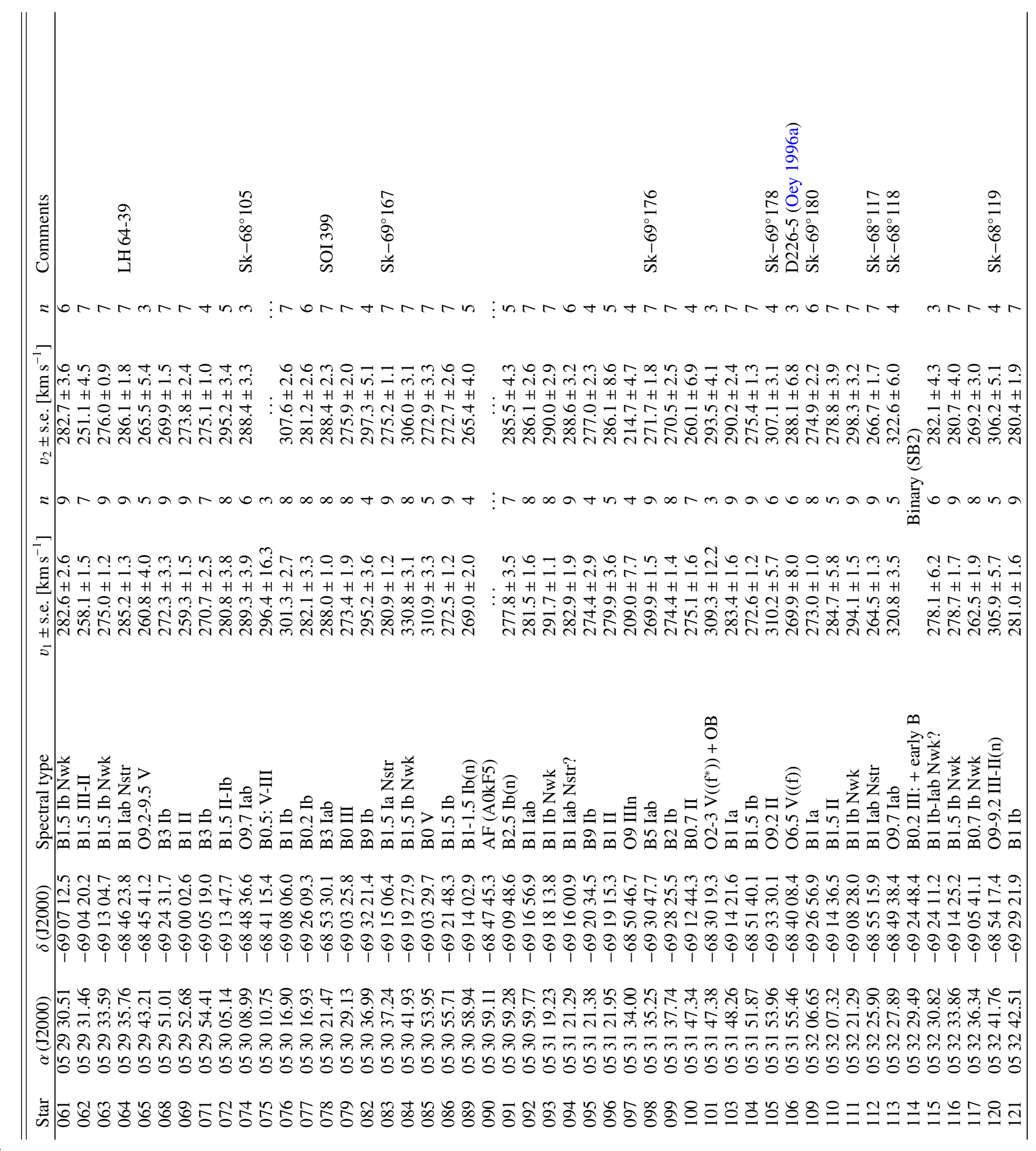




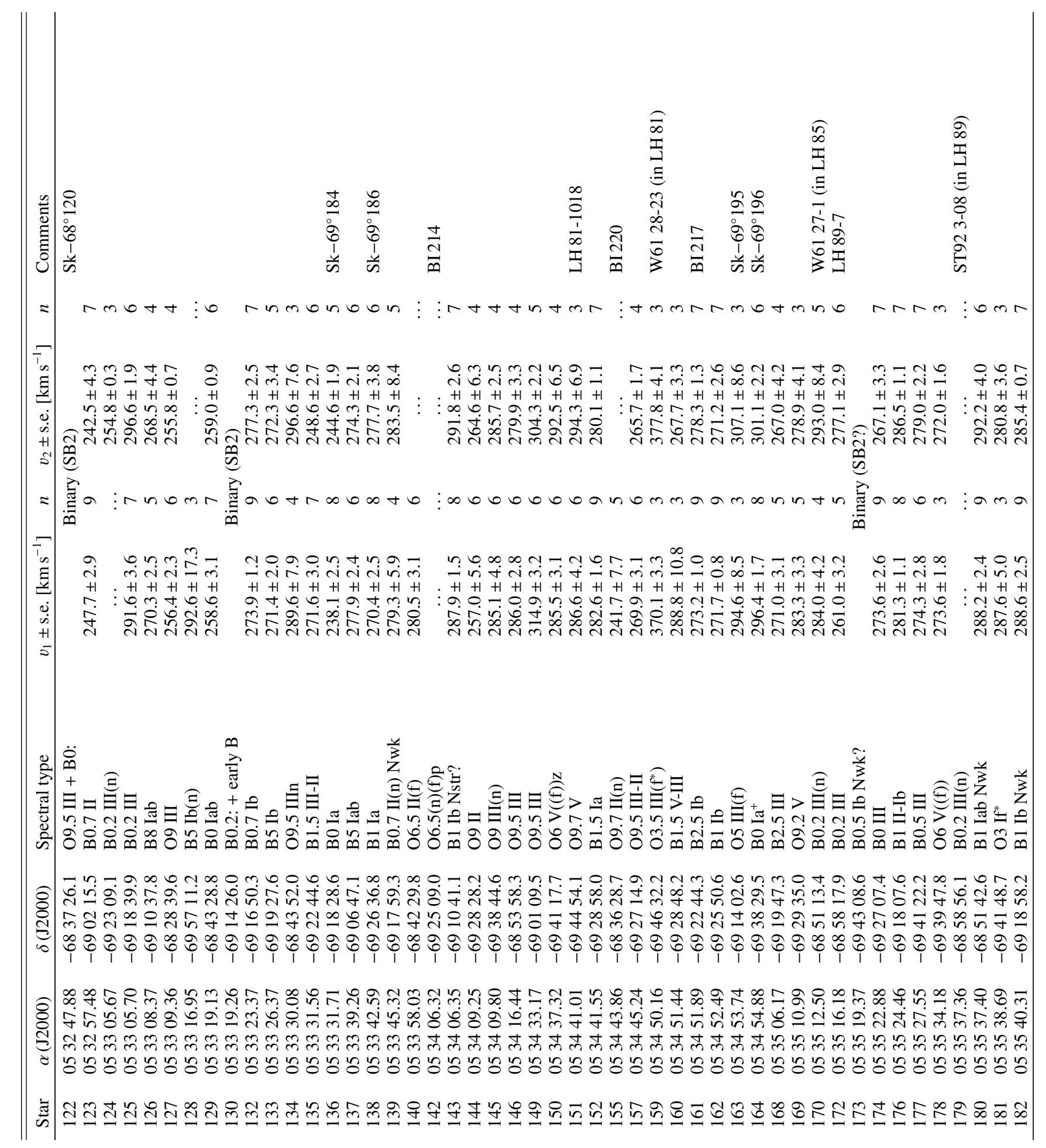




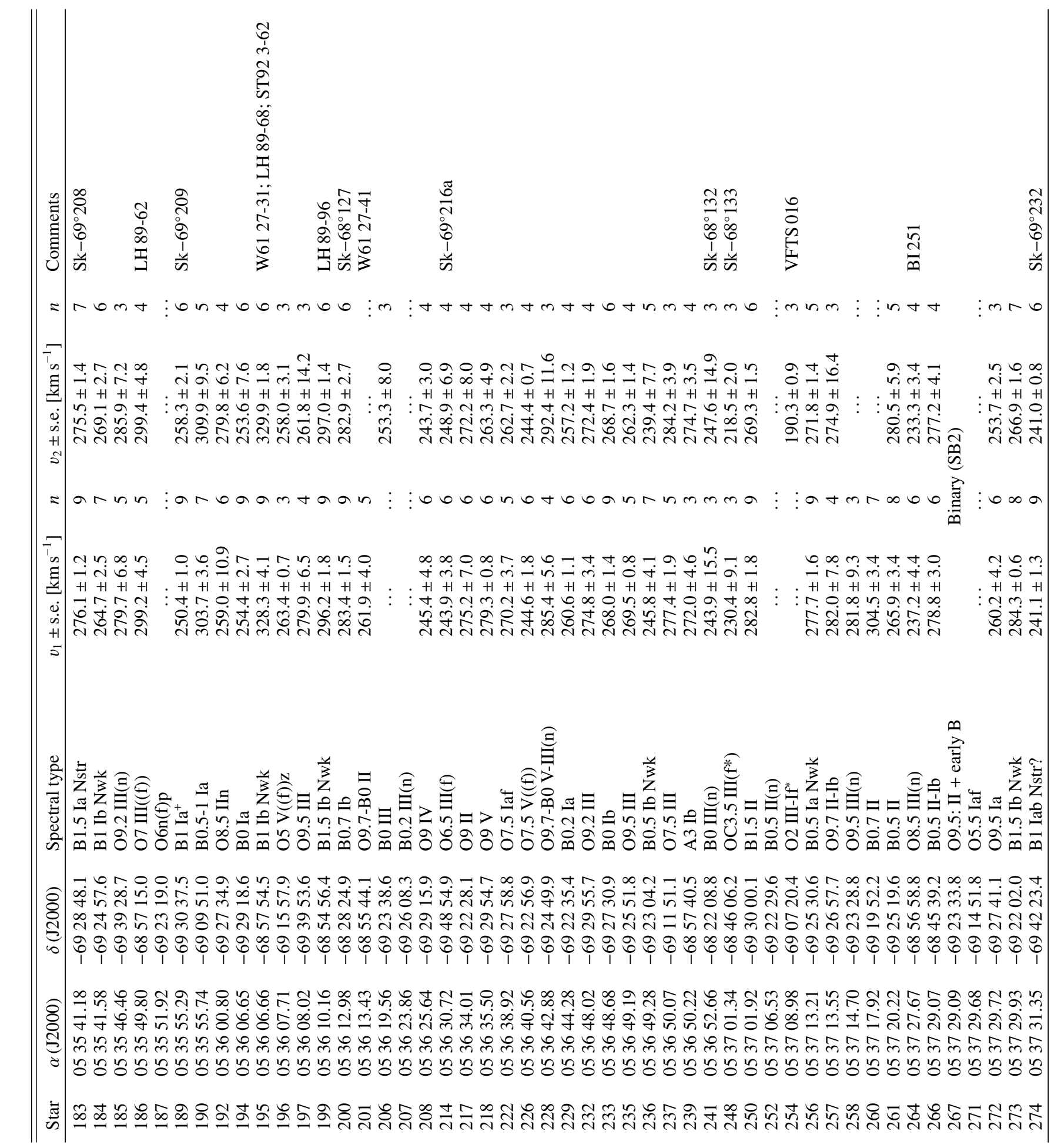


C. J. Evans et al.: AA $\Omega$ spectroscopy of massive stars in the NE of the LMC

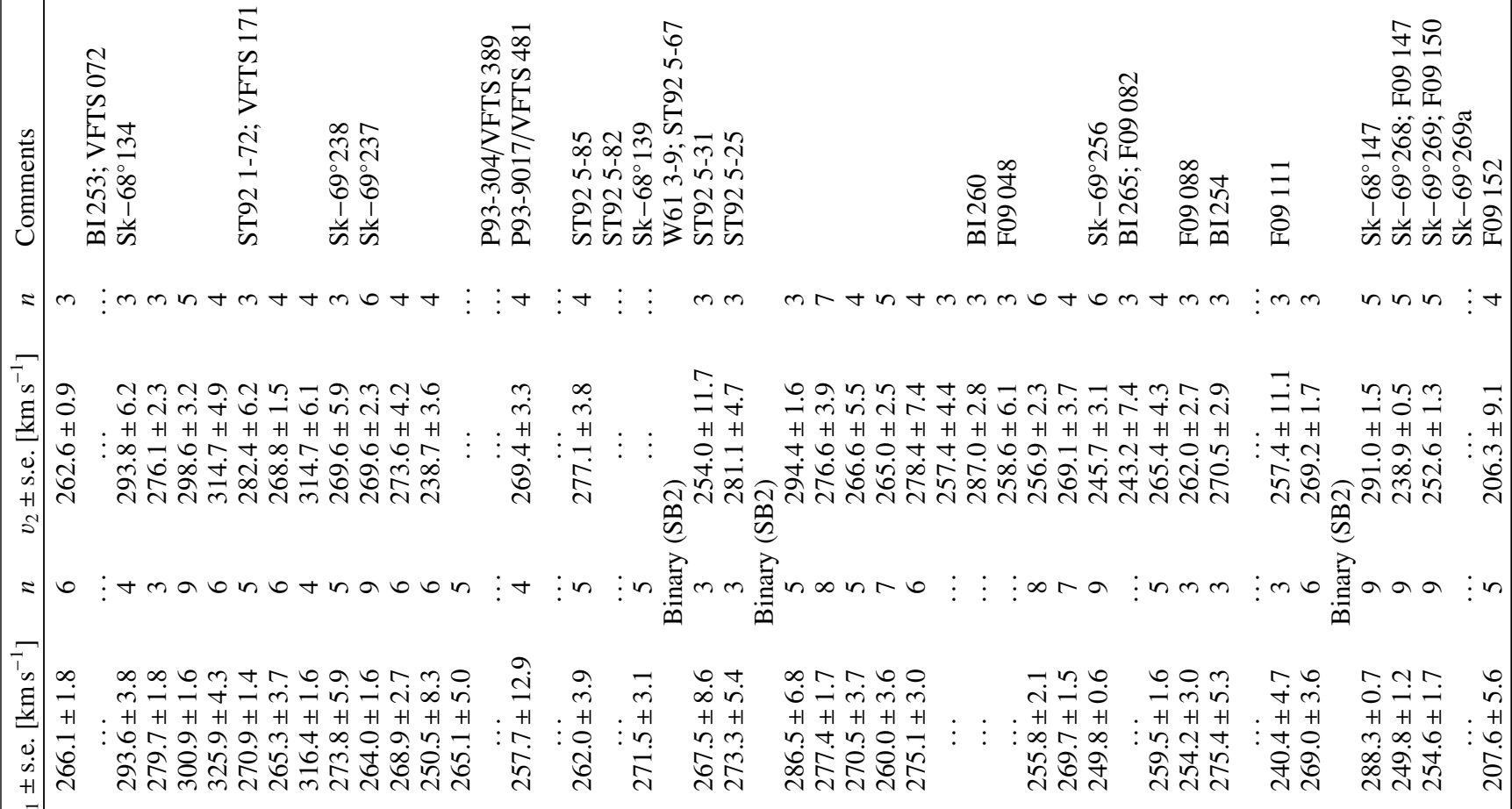

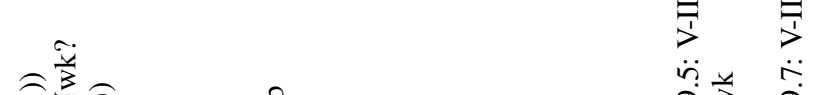

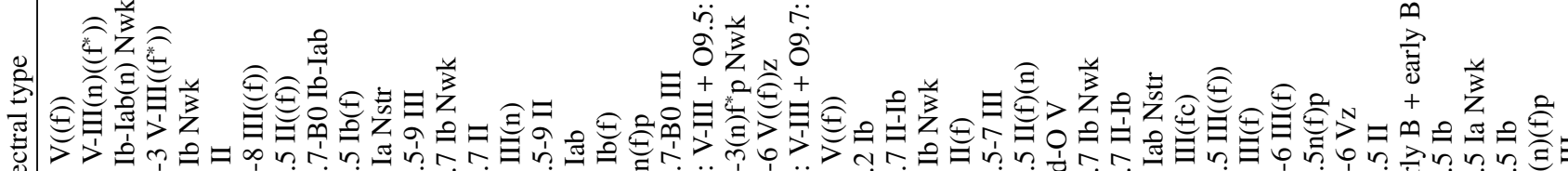

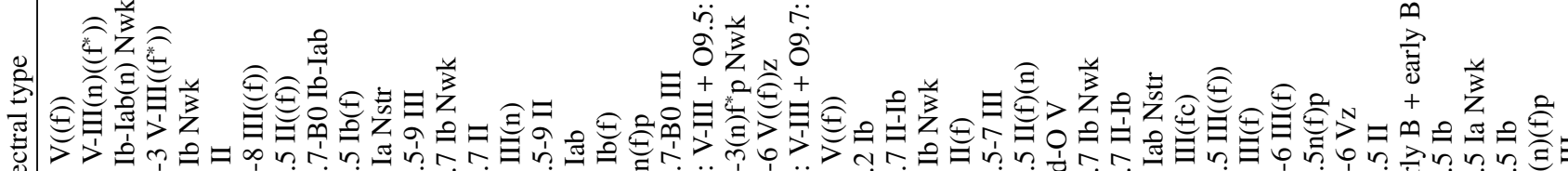

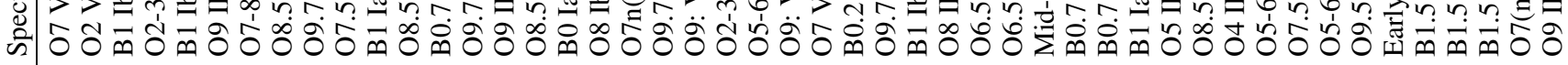

○ी

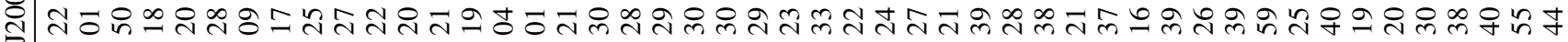

-

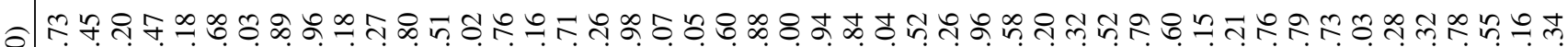

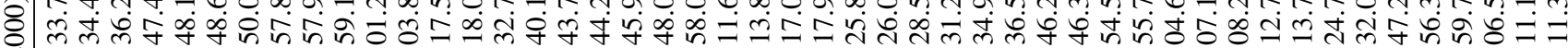

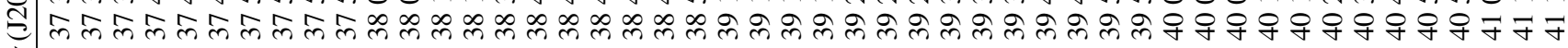

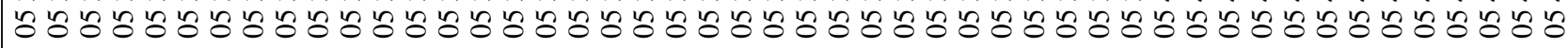

离 


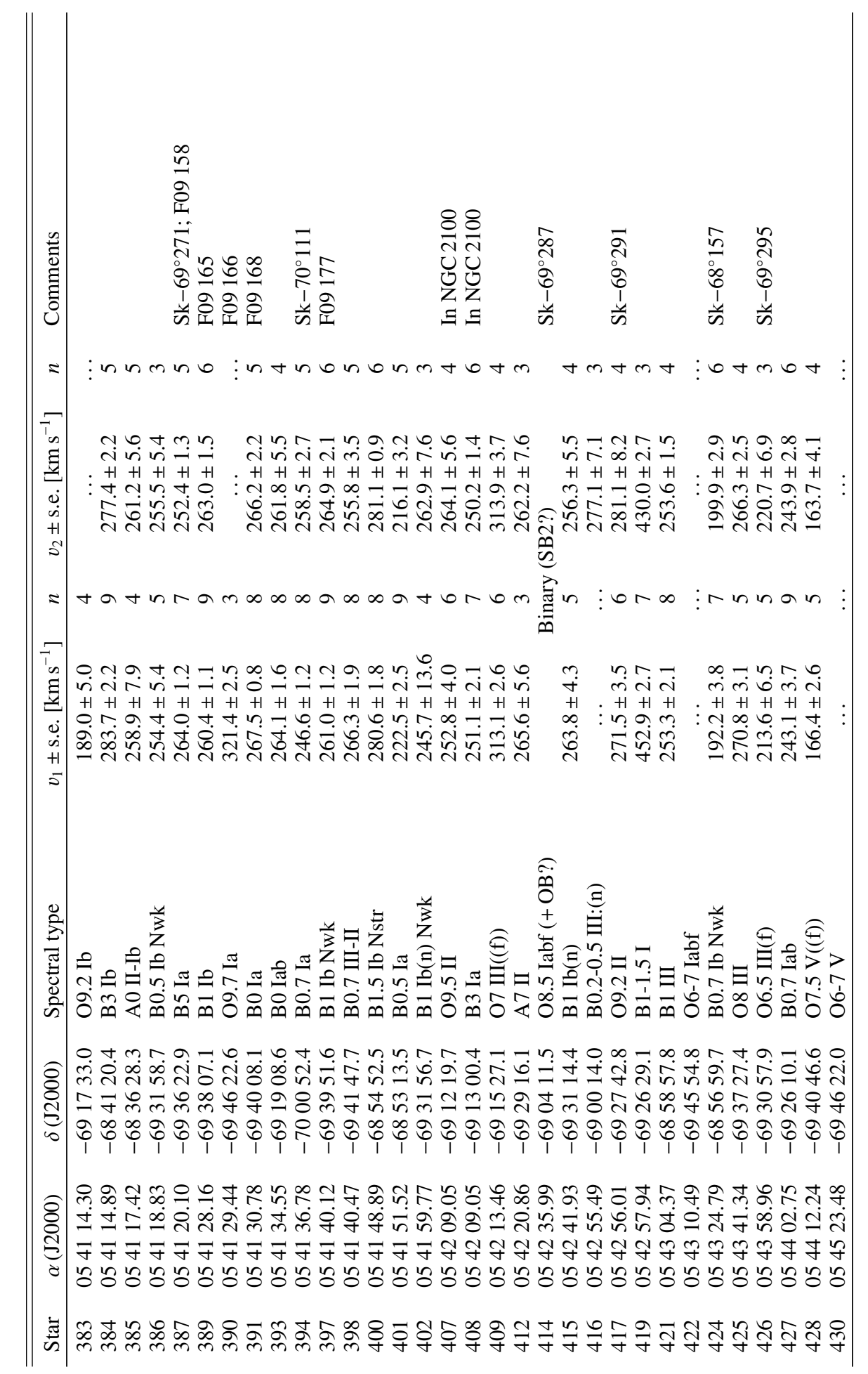


C. J. Evans et al.: AA $\Omega$ spectroscopy of massive stars in the NE of the LMC

Table 3. MCPS photometry of target stars (Zaritsky et al. 2004).

\begin{tabular}{|c|c|c|c|c|c|c|c|}
\hline Star & $U$ & $B$ & $V$ & $I_{\mathrm{c}}$ & $U-B$ & $B-V$ & $V-I_{\mathrm{c}}$ \\
\hline 001 & 12.293 & 13.354 & 13.476 & 13.864 & -1.061 & -0.122 & -0.388 \\
\hline 004 & 12.635 & 13.372 & 13.486 & 13.729 & -0.737 & -0.114 & -0.243 \\
\hline 008 & 12.896 & 14.086 & 13.879 & 14.013 & -1.190 & 0.207 & -0.134 \\
\hline 009 & 12.967 & 13.713 & 13.902 & 14.054 & -0.746 & -0.189 & -0.152 \\
\hline 010 & 12.303 & 13.274 & 13.436 & 13.786 & -0.971 & -0.162 & -0.350 \\
\hline 011 & 12.951 & 13.624 & 13.845 & 13.981 & -0.673 & -0.221 & -0.136 \\
\hline 014 & 11.673 & 12.754 & 12.926 & 13.216 & -1.081 & -0.172 & -0.290 \\
\hline 015 & 11.763 & 12.734 & 12.836 & 13.363 & -0.971 & -0.102 & -0.527 \\
\hline 016 & 12.853 & 13.484 & 13.486 & 13.684 & -0.631 & -0.002 & -0.198 \\
\hline 017 & 12.951 & 13.703 & 13.943 & 13.981 & -0.752 & -0.240 & -0.038 \\
\hline 018 & 13.031 & 13.780 & 13.832 & 13.862 & -0.749 & -0.052 & -0.030 \\
\hline 019 & 12.841 & 13.717 & 13.692 & 13.886 & -0.876 & 0.025 & -0.194 \\
\hline 020 & 11.513 & 12.554 & 12.726 & 13.113 & -1.041 & -0.172 & -0.387 \\
\hline 021 & 12.593 & 13.564 & 06 & 13.801 & -0.971 & -0.142 & -0.095 \\
\hline 024 & 12.263 & 13.264 & 13.406 & 13.537 & -1.001 & -0.142 & -0.131 \\
\hline 025 & 12.323 & 13.304 & 13.426 & 13.508 & -0.981 & -0.122 & -0.082 \\
\hline 026 & 12.483 & 13.364 & 13.436 & 13.553 & -0.881 & -0.072 & -0.117 \\
\hline 027 & 12.633 & 13.624 & 13.756 & 13.879 & -0.991 & -0.132 & -0.123 \\
\hline 028 & 13.132 & 13.850 & 13.926 & 14.049 & -0.718 & -0.076 & -0.123 \\
\hline 030 & 13.088 & 14.152 & 13.853 & 14.285 & -1.064 & 0.299 & -0.432 \\
\hline 033 & 13.332 & 13.767 & 13.821 & 13.951 & -0.435 & -0.054 & -0.130 \\
\hline 034 & 12.243 & 13.254 & 13.356 & 13.431 & -1.011 & -0.102 & -0.075 \\
\hline 036 & 12.889 & 13.823 & 13.883 & 14.101 & -0.934 & -0.060 & -0.218 \\
\hline 038 & 12.663 & 13.939 & 13.720 & 14.064 & -1.276 & 0.219 & -0.344 \\
\hline 040 & 12.283 & 13.444 & 13.666 & 13.854 & -1.161 & -0.222 & -0.188 \\
\hline 042 & 13.039 & 13.682 & 13.804 & 13.857 & -0.643 & -0.122 & -0.053 \\
\hline 043 & 12.273 & 13.204 & & 13.741 & -0.931 & -0.062 & -0.475 \\
\hline 045 & 13.361 & 14.071 & 13. & 13.938 & -0 & 0.291 & -0.158 \\
\hline 047 & 12.8 & 13 & 6 & 13.2 & -0 & -0.002 & -0.078 \\
\hline 049 & 13.725 & 13.726 & 13. & 13.558 & -0 . & 0.253 & -0.085 \\
\hline 050 & 12.658 & 13.603 & 13.644 & 13.873 & -0.945 & -0.041 & -0.229 \\
\hline 053 & 12.491 & 13.511 & 13.534 & 13.829 & -1.020 & -0.023 & -0.295 \\
\hline 054 & 12.636 & 13.743 & 13.587 & 13.651 & -1.107 & 0.156 & -0.064 \\
\hline 055 & 12.995 & 13.633 & 13.652 & 13.685 & -0.638 & -0.019 & -0.033 \\
\hline 056 & 12.543 & 13.474 & 13.576 & 13.812 & -0.931 & -0.102 & -0.236 \\
\hline 057 & 12.353 & 13.444 & 13.656 & 13.816 & -1.091 & -0.212 & -0.160 \\
\hline 058 & 12.975 & 13.737 & 13.785 & 14.546 & -0.762 & -0.048 & -0.761 \\
\hline 059 & 13.059 & 13.869 & 13.858 & 14.169 & -0.810 & 0.011 & -0.311 \\
\hline 060 & 11.453 & 12.444 & 12.546 & 12.673 & -0.991 & -0.102 & -0.127 \\
\hline 061 & 12.503 & 13.434 & 13.546 & 13.603 & -0.931 & -0.112 & -0.057 \\
\hline 062 & 13.013 & 13.654 & 13.778 & 13.906 & -0.641 & -0.124 & -0.128 \\
\hline 063 & 12.944 & 13.756 & 01 & 13.985 & -0.812 & -0.045 & -0.184 \\
\hline 064 & 12.303 & 13 & & & -0.961 & 12 & -0.124 \\
\hline 0 & 12 & & & & -1 & -0 & -0.216 \\
\hline 068 & 13.296 & 13.937 & 13.9 & 14.2 & -0 . & -0 & -0.268 \\
\hline 069 & 13.026 & 13.752 & 13.928 & 14.082 & -0.726 & -0.176 & -0.154 \\
\hline 071 & 13.230 & 13.844 & 13.841 & 13.902 & -0.614 & 0.003 & -0.061 \\
\hline 072 & 12.622 & 13.505 & 13.592 & 13.705 & -0.883 & -0.087 & -0.113 \\
\hline 074 & 11.903 & 12.974 & 13.116 & 13.259 & -1.071 & -0.142 & -0.143 \\
\hline 075 & 12.836 & 13.709 & 13.888 & 13.908 & -0.873 & -0.179 & -0.020 \\
\hline 076 & 12.987 & 13.787 & 13.858 & 13.953 & -0.800 & -0.071 & -0.095 \\
\hline 077 & 12.797 & 13.708 & 13.878 & 14.143 & -0.911 & -0.170 & -0.265 \\
\hline 078 & 12.293 & 13.094 & 13.186 & 13.210 & -0.801 & -0.092 & -0.024 \\
\hline 079 & 12.824 & 13.676 & 13.842 & 14.043 & -0.852 & -0.166 & -0.201 \\
\hline 082 & 13.710 & 14.014 & 13.969 & 13.985 & -0.304 & 0.045 & -0.016 \\
\hline 083 & 11.343 & 12.304 & 12.386 & 12.510 & -0.961 & -0.082 & -0.124 \\
\hline 084 & 12.743 & 13.674 & 13.726 & 14.021 & -0.931 & -0.052 & -0.295 \\
\hline 085 & 12.623 & 13.634 & 13.816 & 13.937 & -1.011 & -0.182 & -0.121 \\
\hline 086 & 12.513 & 13.434 & 13.546 & 13.884 & -0.921 & -0.112 & -0.338 \\
\hline 089 & 13.030 & 13.858 & 13.957 & 13.965 & -0.828 & -0.099 & -0.008 \\
\hline 090 & 14.383 & 14.274 & 13.896 & 14.359 & 0.109 & 0.378 & -0.463 \\
\hline 091 & 12.583 & 13.494 & 13.596 & 13.687 & -0.911 & -0.102 & -0.091 \\
\hline 092 & 12.043 & 13.024 & 13.136 & 13.290 & -0.981 & -0.112 & -0.154 \\
\hline 0 & 12.675 & 13.701 & 13.700 & 13.883 & -1.026 & 0.001 & -0.183 \\
\hline 09 & 12.323 & 13.314 & 13.436 & 13.590 & -0.991 & -0.122 & -0.154 \\
\hline 095 & 13.302 & 13.717 & 13.643 & 13.677 & -0.415 & 0.074 & -0.034 \\
\hline
\end{tabular}


A\&A 584, A5 (2015)

Table 3. continued.

\begin{tabular}{|c|c|c|c|c|c|c|c|}
\hline Star & $U$ & $B$ & $V$ & $I_{\mathrm{c}}$ & $U-B$ & $B-V$ & $V-I_{\mathrm{c}}$ \\
\hline 096 & 12.924 & 13.752 & 13.894 & 14.073 & -0.828 & -0.142 & -0.179 \\
\hline 097 & 12.799 & 13.709 & 13.872 & 14.172 & -0.910 & -0.163 & -0.300 \\
\hline 098 & 12.053 & 12.814 & 12.816 & 12.830 & -0.761 & -0.002 & -0.014 \\
\hline 099 & 12.673 & 13.544 & 13.646 & 13.722 & -0.871 & -0.102 & -0.076 \\
\hline 100 & 12.802 & 13.590 & 13.784 & 13.931 & -0.788 & -0.194 & -0.147 \\
\hline 101 & 13.039 & 14.082 & 13.997 & 14.090 & -1.043 & 0.085 & -0.093 \\
\hline 103 & 11.883 & 12.854 & 12.956 & 13.002 & -0.971 & -0.102 & -0.046 \\
\hline 104 & 12.744 & 13.897 & 13.647 & 13.778 & -1.153 & 0.250 & -0.131 \\
\hline 105 & 12.130 & 13.173 & 13.257 & 13.417 & -1.043 & -0.084 & -0.160 \\
\hline 106 & 12.473 & 13.444 & 13.566 & 13.650 & -0.971 & -0.122 & -0.084 \\
\hline 109 & 11.853 & 12.844 & 12.976 & 13.134 & -0.991 & -0.132 & -0.158 \\
\hline 110 & 13.023 & 13.692 & 13.748 & 13.992 & -0.669 & -0.056 & -0.244 \\
\hline 111 & 13.024 & 13.603 & 13.805 & 13.981 & -0.579 & -0.202 & -0.176 \\
\hline 112 & 12.443 & 13.414 & 26 & 13.552 & -0.971 & -0.112 & .026 \\
\hline 113 & 11.703 & 12.774 & 12.916 & 13.088 & -1.071 & -0.142 & -0.172 \\
\hline 114 & 12.968 & 13.802 & 13.885 & 14.177 & -0.834 & -0.083 & -0.292 \\
\hline 115 & 12.413 & 13.414 & 13.526 & 13.689 & -1.001 & -0.112 & -0.163 \\
\hline 116 & 12.313 & 13.274 & 13.356 & 13.539 & -0.961 & -0.082 & -0.183 \\
\hline 117 & 12.884 & 13.708 & 13.769 & 13.835 & -0.824 & -0.061 & -0.066 \\
\hline 120 & 12.473 & 13.534 & 13.676 & 13.758 & -1.061 & -0.142 & -0.082 \\
\hline 121 & 12.243 & 13.204 & 13.316 & 13.459 & -0.961 & -0.112 & -0.143 \\
\hline 122 & 12.123 & 13.204 & 13.416 & 13.435 & -1.081 & -0.212 & -0.019 \\
\hline 123 & 13.175 & 13.899 & 13.901 & 13.948 & -0.724 & -0.002 & -0.047 \\
\hline 124 & 12.980 & 13.851 & 13.935 & 14.164 & -0.871 & -0.084 & -0.229 \\
\hline 125 & 12.956 & 13.845 & 13.957 & 14.242 & -0.889 & -0.112 & -0.285 \\
\hline 126 & 13.237 & 13.676 & 580 & 14.436 & -0.439 & 0.096 & -0.856 \\
\hline 127 & 12.760 & 13.795 & 92 & 17 & -1.035 & -0.097 & .225 \\
\hline 128 & 13.6 & 95 & & 07 & -0 & 43 & 155 \\
\hline 129 & 12.113 & 134 & 13 & 13.355 & -1 . & -0 & -0.109 \\
\hline 130 & 12.163 & 13.144 & 13.246 & 13.522 & -0 & -0.102 & -0.276 \\
\hline 132 & 12.513 & 13.494 & 13.586 & 13.647 & -0.981 & -0.092 & -0.061 \\
\hline 133 & 13.109 & 13.689 & 13.623 & 13.738 & -0.580 & 0.066 & -0.115 \\
\hline 134 & 12.774 & 13.808 & 13.947 & 14.051 & -1.034 & -0.139 & -0.104 \\
\hline 135 & 12.843 & 13.582 & 13.561 & 13.868 & -0.739 & 0.021 & -0.307 \\
\hline 136 & 11.923 & 12.924 & 13.046 & 13.161 & -1.001 & -0.122 & -0.115 \\
\hline 137 & 12.543 & 13.254 & 13.156 & 13.183 & -0.711 & 0.098 & -0.027 \\
\hline 138 & 11.943 & 12.934 & 13.026 & 13.132 & -0.991 & -0.092 & -0.106 \\
\hline 139 & 12.799 & 13.642 & 13.619 & 13.906 & -0.843 & 0.023 & -0.287 \\
\hline 140 & 12.313 & 13.384 & 13.536 & 13.638 & -1.071 & -0.152 & -0.102 \\
\hline 142 & 12.003 & 13.084 & 13.236 & 13.411 & -1.081 & -0.152 & -0.175 \\
\hline 143 & & 174 & 566 & 06 & -0.911 & -0.092 & -0.040 \\
\hline 144 & & 54 & & 47 & 71 & 152 & 141 \\
\hline 145 & 12 & 94 & 27 & 10 & -0 & 33 & 313 \\
\hline 146 & 12.860 & 14.104 & 13.930 & 14.089 & -1.244 & 0.174 & -0.159 \\
\hline 149 & 12.940 & 13.791 & 13.872 & 13.995 & -0.851 & -0.081 & -0.123 \\
\hline 150 & 13.009 & 13.864 & 13.986 & 14.342 & -0.855 & -0.122 & -0.356 \\
\hline 151 & 12.954 & 13.603 & 13.743 & 13.927 & -0.649 & -0.140 & -0.184 \\
\hline 152 & 11.275 & 11.910 & 11.417 & 12.432 & -0.635 & 0.493 & -1.015 \\
\hline 15 & 12.722 & 13.710 & 13.853 & 14.098 & -0.988 & -0.143 & -0.245 \\
\hline 157 & 12.737 & 13.616 & 13.793 & 14.023 & -0.879 & -0.177 & -0.230 \\
\hline 159 & 12.698 & 13.596 & 13.702 & 13.752 & -0.898 & -0.106 & -0.050 \\
\hline 160 & 14.277 & 14.011 & 13.695 & 15.370 & 0.266 & 0.316 & -1.675 \\
\hline 161 & 12.563 & 13.384 & 13.416 & 13.500 & -0.821 & -0.032 & -0.084 \\
\hline 162 & 12.533 & 13.464 & 13.506 & 13.764 & -0.931 & -0.042 & -0.258 \\
\hline 163 & 12.193 & 13.234 & 13.366 & 13.412 & -1.041 & -0.132 & -0.046 \\
\hline 164 & 11.323 & 12.163 & 59 & 12.545 & -0.840 & 0.004 & -0.386 \\
\hline 168 & 12.313 & 13.084 & 13.136 & 14.399 & -0.771 & -0.052 & -1.263 \\
\hline 169 & 12.734 & 13.638 & 13.807 & 14.026 & -0.904 & -0.169 & -0.219 \\
\hline 170 & 12.820 & 13.533 & 13.752 & 13.969 & -0.713 & -0.219 & -0.217 \\
\hline 172 & 13.074 & 13.835 & 13.917 & 14.015 & -0.761 & -0.082 & -0.098 \\
\hline 173 & 12.749 & 12.658 & 12.518 & 12.835 & 0.091 & 0.140 & -0.317 \\
\hline 174 & 12.339 & 13.188 & 13.287 & 13.544 & -0.849 & -0.099 & -0.257 \\
\hline 176 & 13.341 & 14.071 & 13.940 & 14.025 & -0.730 & 0.131 & -0.085 \\
\hline 177 & 13.119 & 13.866 & 13.922 & 14.162 & -0.747 & -0.056 & -0.240 \\
\hline 178 & 12.507 & 13.349 & 13.297 & 13.726 & -0.842 & 0.052 & -0.429 \\
\hline 179 & 13.308 & 14.121 & 13.634 & 13.968 & -0.813 & 0.487 & -0.334 \\
\hline 180 & 11.963 & 12.924 & 13.056 & 13.175 & -0.961 & -0.132 & -0.119 \\
\hline
\end{tabular}


Table 3. continued.

\begin{tabular}{|c|c|c|c|c|c|c|c|}
\hline Star & $U$ & $B$ & $V$ & $I_{\mathrm{c}}$ & $U-B$ & $B-V$ & $V-I_{\mathrm{c}}$ \\
\hline 181 & 12.335 & 13.139 & 13.280 & 13.514 & -0.804 & -0.141 & -0.234 \\
\hline 182 & 13.204 & 13.868 & 13.887 & 14.062 & -0.664 & -0.019 & -0.175 \\
\hline 183 & 11.823 & 12.784 & 12.856 & 12.893 & -0.961 & -0.072 & -0.037 \\
\hline 184 & 12.951 & 13.743 & 13.824 & 14.065 & -0.792 & -0.081 & -0.241 \\
\hline 185 & 12.713 & 13.247 & 13.251 & 13.536 & -0.534 & -0.004 & -0.285 \\
\hline 186 & 13.263 & 13.892 & 13.948 & 13.989 & -0.629 & -0.056 & -0.041 \\
\hline 187 & 12.113 & 13.214 & 13.396 & 13.481 & -1.101 & -0.182 & -0.085 \\
\hline 189 & 10.981 & 11.735 & 11.567 & 11.833 & -0.754 & 0.168 & -0.266 \\
\hline 190 & 11.163 & 11.904 & 11.606 & 12.880 & -0.741 & 0.298 & -1.274 \\
\hline 192 & 12.579 & 13.507 & 13.520 & 13.809 & -0.928 & -0.013 & -0.289 \\
\hline 194 & 11.993 & 13.064 & 13.086 & 13.124 & -1.071 & -0.022 & -0.038 \\
\hline 195 & 13.141 & 13.846 & 13.827 & 13.910 & -0.705 & 0.019 & -0.083 \\
\hline 196 & 12.948 & 13.905 & 13.870 & 14.117 & -0.957 & 0.035 & -0.247 \\
\hline 197 & 14.084 & 13.274 & 13.386 & 13.517 & 0.810 & -0.112 & -0.131 \\
\hline 199 & 13.254 & 13.897 & & 14.019 & -0.643 & -0.093 & -0.029 \\
\hline 200 & 12.593 & 13.604 & 13.726 & 13.896 & -1.011 & -0.122 & -0.170 \\
\hline 201 & 13.092 & 13.894 & 13.990 & 14.204 & -0.802 & -0.096 & -0.214 \\
\hline 206 & 12.840 & 13.697 & 13.775 & 13.904 & -0.857 & -0.078 & -0.129 \\
\hline 207 & 13.062 & 13.957 & 13.937 & 14.334 & -0.895 & 0.020 & -0.397 \\
\hline 208 & 11.923 & 12.944 & 13.016 & 13.456 & -1.021 & -0.072 & -0.440 \\
\hline 214 & 12.573 & 13.316 & 13.613 & 13.772 & -0.743 & -0.297 & -0.159 \\
\hline 217 & 12.273 & 13.304 & 13.426 & 13.473 & -1.031 & -0.122 & -0.047 \\
\hline 218 & 12.750 & 13.667 & 13.704 & 14.110 & -0.917 & -0.037 & -0.406 \\
\hline 222 & 11.573 & 12.614 & 12.726 & 12.928 & -1.041 & -0.112 & -0.202 \\
\hline 226 & 12.836 & 13.833 & 13.847 & 14.080 & -0.997 & -0.014 & -0.233 \\
\hline 228 & 13.016 & 13.890 & 13.936 & 14.262 & -0.874 & -0.046 & -0.326 \\
\hline 229 & 12.073 & 13.074 & & 13.264 & -1.001 & -0.082 & -0.108 \\
\hline 232 & 12.330 & 13.163 & & 13.433 & -0.833 & -0.003 & -0.267 \\
\hline 233 & 13.146 & 14.000 & 13. & 14.301 & -0.854 & 0.019 & -0.320 \\
\hline 235 & 12.133 & 13.124 & 13. & 13.785 & -0.991 & -0.182 & -0.479 \\
\hline 236 & 12.969 & 13.819 & 13. & 14.049 & -0.850 & -0.098 & -0.132 \\
\hline 237 & 12.493 & 13.494 & 13.506 & 13.515 & -1.001 & -0.012 & -0.009 \\
\hline 239 & 14.003 & 13.874 & 13.546 & 13.599 & 0.129 & 0.328 & -0.053 \\
\hline 241 & 12.811 & 13.799 & 13.939 & 14.169 & -0.988 & -0.140 & -0.230 \\
\hline 248 & 12.023 & 13.084 & 13.206 & 13.209 & -1.061 & -0.122 & -0.003 \\
\hline 250 & 12.843 & 13.653 & 13.599 & 13.991 & -0.810 & 0.054 & -0.392 \\
\hline 252 & 12.859 & 13.693 & 13.592 & 13.747 & -0.834 & 0.101 & -0.155 \\
\hline 254 & 12.623 & 13.584 & 13.546 & 13.816 & -0.961 & 0.038 & -0.270 \\
\hline 256 & 12.766 & 13.555 & 13.656 & 13.704 & -0.789 & -0.101 & -0.048 \\
\hline 257 & 12.353 & 13.344 & 13.416 & 13.553 & -0.991 & -0.072 & -0.137 \\
\hline 258 & 12.972 & 13.812 & 13.742 & 13.993 & -0.840 & 0.070 & -0.251 \\
\hline 260 & 13.018 & 13.741 & & 13.870 & -0.723 & 0.067 & -0.196 \\
\hline 261 & & 13 & & 4 & -0 & -0 & -0.091 \\
\hline 264 & 12 & 13 & 13. & 2 & -0.722 & -0 & -0.055 \\
\hline 266 & 13.020 & 13.925 & 13.9 & 13.981 & -0.905 & 0.008 & -0.064 \\
\hline 267 & 12.223 & 13.204 & 13.356 & 13.423 & -0.981 & -0.152 & -0.067 \\
\hline 271 & 12.493 & 13.464 & 13.486 & 13.651 & -0.971 & -0.022 & -0.165 \\
\hline 272 & 11.713 & 12.744 & 12.826 & 12.929 & -1.031 & -0.082 & -0.103 \\
\hline 273 & 12.663 & 13.544 & 13.516 & 13.539 & -0.881 & 0.028 & -0.023 \\
\hline 274 & 12.467 & 13.207 & 13.241 & 13.265 & -0.740 & -0.034 & -0.024 \\
\hline 275 & 12.868 & 13.880 & 13.984 & 14.166 & -1.012 & -0.104 & -0.182 \\
\hline 276 & 12.765 & 13.650 & 13.669 & 13.742 & -0.885 & -0.019 & -0.073 \\
\hline 277 & 11.818 & 13.655 & 13.656 & 13.696 & -1.837 & -0.001 & -0.040 \\
\hline 280 & 12.990 & 13.908 & 13.789 & 13.803 & -0.918 & 0.119 & -0.014 \\
\hline 281 & 12.860 & 13.760 & 13.655 & 13.895 & -0.900 & 0.105 & -0.240 \\
\hline 282 & 12.660 & 13.567 & 13.658 & 13.838 & -0.907 & -0.091 & -0.180 \\
\hline 283 & 13.096 & 13.699 & 13.840 & 13.900 & -0.603 & -0.141 & -0.060 \\
\hline 284 & 12.917 & 13.787 & 13.724 & 13.780 & -0.870 & 0.063 & -0.056 \\
\hline 285 & 12.283 & 13.234 & 13.276 & 13.425 & -0.951 & -0.042 & -0.149 \\
\hline 286 & 11.212 & 12.069 & 12.267 & 12.370 & -0.857 & -0.198 & -0.103 \\
\hline 287 & 11.243 & 12.122 & 12.032 & 12.107 & -0.879 & 0.090 & -0.075 \\
\hline 288 & 13.065 & 13.955 & 13.980 & 14.185 & -0.890 & -0.025 & -0.205 \\
\hline 290 & 13.146 & 13.927 & 13.684 & 13.742 & -0.781 & 0.243 & -0.058 \\
\hline 291 & 13.227 & 14.056 & 13.920 & 14.168 & -0.829 & 0.136 & -0.248 \\
\hline 296 & 12.123 & 13.084 & 12.916 & 13.727 & -0.961 & 0.168 & -0.811 \\
\hline 308 & 12.901 & 14.010 & 13.905 & 14.010 & -1.109 & 0.105 & -0.105 \\
\hline 315 & 12.393 & 13.384 & 13.466 & 13.556 & -0.991 & -0.082 & -0.090 \\
\hline
\end{tabular}


A\&A 584, A5 (2015)

Table 3. continued.

\begin{tabular}{|c|c|c|c|c|c|c|c|}
\hline Star & $U$ & $B$ & $V$ & $I_{\mathrm{c}}$ & $U-B$ & $B-V$ & $V-I_{\mathrm{c}}$ \\
\hline 318 & 12.749 & 13.702 & 13.700 & 13.773 & -0.953 & 0.002 & -0.073 \\
\hline 320 & 12.824 & 13.794 & 13.746 & 13.945 & -0.970 & 0.048 & -0.199 \\
\hline 324 & 12.713 & 13.637 & 13.801 & 14.087 & -0.924 & -0.164 & -0.286 \\
\hline 330 & 12.832 & 13.955 & 13.740 & 14.177 & -1.123 & 0.215 & -0.437 \\
\hline 333 & 11.415 & 12.374 & 12.273 & 12.275 & -0.959 & 0.101 & -0.002 \\
\hline 334 & 12.810 & 13.966 & 13.551 & 14.168 & -1.156 & 0.415 & -0.617 \\
\hline 337 & 13.008 & 13.824 & 13.900 & 14.027 & -0.816 & -0.076 & -0.127 \\
\hline 338 & 12.712 & 13.736 & 13.519 & 13.855 & -1.024 & 0.217 & -0.336 \\
\hline 339 & 12.912 & 13.767 & 13.821 & 13.891 & -0.855 & -0.054 & -0.070 \\
\hline 340 & 11.973 & 13.034 & 13.036 & 13.165 & -1.061 & -0.002 & -0.129 \\
\hline 341 & 13.074 & 14.352 & 12.599 & 14.878 & -1.278 & 1.753 & -2.279 \\
\hline 343 & 14.855 & 13.826 & 13.771 & 13.985 & 1.029 & 0.055 & -0.214 \\
\hline 344 & 13.426 & 13.883 & 13.670 & 13.817 & -0.457 & 0.213 & -0.147 \\
\hline 345 & 12.635 & 13.569 & 13.522 & 13.664 & -0.934 & 0.047 & -0.142 \\
\hline 352 & 13.216 & 14.168 & 13.787 & 14.096 & -0 & 0.381 & 309 \\
\hline 353 & 13.225 & 14.048 & 13.924 & 14.113 & -0.823 & 0.124 & -0.189 \\
\hline 355 & 13.000 & 13.895 & 13.917 & 14.161 & -0.895 & -0.022 & -0.244 \\
\hline 356 & 11.713 & 12.644 & 12.666 & 12.772 & -0.931 & -0.022 & -0.106 \\
\hline 361 & 11.526 & 12.346 & 12.375 & 12.493 & -0.820 & -0.029 & -0.118 \\
\hline 362 & 12.883 & 13.842 & 13.956 & 14.088 & -0.959 & -0.114 & -0.132 \\
\hline 364 & 12.867 & 13.562 & 13.629 & 13.637 & -0.695 & -0.067 & -0.008 \\
\hline 367 & 13.029 & 13.730 & 13.892 & 14.087 & -0.701 & -0.162 & -0.195 \\
\hline 368 & 12.113 & 13.174 & 13.296 & 13.356 & -1.061 & -0.122 & -0.060 \\
\hline 369 & 12.013 & 12.820 & 12.365 & 12.638 & -0.807 & 0.455 & -0.273 \\
\hline 371 & 12.805 & 13.626 & 13.525 & 13.651 & -0.821 & 0.101 & -0.126 \\
\hline 374 & 13.480 & 13.934 & 13.761 & 14.151 & -0.454 & 0.173 & -0.390 \\
\hline 375 & 11.923 & 12.894 & 12.966 & 13.088 & -0 & -0.072 & -0.122 \\
\hline 376 & 11.803 & 12.445 & 12.242 & 12.308 & -0. & 0.203 & -0.066 \\
\hline 377 & 12.839 & 13 & 13 & & -0 . & 0.085 & -0.111 \\
\hline 380 & 12.573 & 6 & 14 & 1 & -0 . & -0.028 & -0.113 \\
\hline 381 & 12.985 & 8 & 13. & 1 & -0 & -0.113 & -0 \\
\hline 383 & 12.523 & 13.534 & 13.646 & 7 & -1 . & -0.112 & -0.151 \\
\hline 384 & 12.959 & 13.698 & 13.628 & 1 & -0.739 & 0.070 & -0.043 \\
\hline 385 & 13.672 & 13.859 & 13.799 & 13.809 & -0.187 & 0.060 & -0.010 \\
\hline 386 & 12.881 & 13.777 & 13.658 & 13.896 & -0.896 & 0.119 & -0.238 \\
\hline 387 & 11.307 & 11.790 & 11.790 & 11.910 & -0.483 & 0.000 & -0.120 \\
\hline 389 & 12.137 & 12.410 & 12.848 & 13.071 & -0.273 & -0.438 & -0.223 \\
\hline 390 & 11.515 & 12.170 & 12.285 & 12.303 & -0.655 & -0.115 & -0.018 \\
\hline 391 & 11.673 & 12.479 & 12.584 & 12.945 & -0.806 & -0.105 & -0.361 \\
\hline 393 & 12.143 & 13.154 & 13.296 & 13.406 & -1.011 & -0.142 & -0.110 \\
\hline 394 & 10.812 & 11.872 & 11.516 & 1.965 & -1.060 & 0.356 & -0.449 \\
\hline 397 & 12.795 & 13.507 & 13.693 & 13.755 & -0.712 & -0.186 & -0.062 \\
\hline 398 & 13.011 & 13.739 & 83 & 41 & -0.728 & -0 . & -0.158 \\
\hline 400 & 12 & 4 & & 7 & -0 & -0 & -0.091 \\
\hline 401 & 11.963 & 12.9 & 13.0 & 4 & -0 & -0 & -0 \\
\hline 02 & 13.297 & 14.086 & 13.795 & 14.045 & -0.789 & 0.291 & -0.250 \\
\hline 07 & 12.253 & 13.114 & 13.156 & 13.545 & -0.861 & -0.042 & -0.389 \\
\hline 408 & 11.893 & 12.614 & 12.566 & 12.919 & -0.721 & 0.048 & -0.353 \\
\hline 409 & 11.923 & 13.024 & 13.206 & 13.372 & -1.101 & -0.182 & -0.166 \\
\hline 412 & 11.453 & 12.354 & 12.496 & 13.112 & -0.901 & -0.142 & -0.616 \\
\hline 414 & 11.333 & 12.224 & 12.216 & 14.113 & -0.891 & 0.008 & -1.897 \\
\hline 415 & 13.139 & 13.961 & 13.841 & 13.912 & -0.822 & 0.120 & -0.071 \\
\hline 416 & 13.225 & 13.795 & 13.954 & 13.988 & -0.570 & -0.159 & -0.034 \\
\hline 417 & 11.653 & 12.664 & 12.696 & 13.130 & -1.011 & -0.032 & -0.434 \\
\hline 419 & 12.263 & 13.234 & 13.256 & 13.354 & -0.971 & -0.022 & -0.098 \\
\hline 421 & 13.079 & 13.672 & 13.703 & 14.008 & -0.593 & -0.031 & -0.305 \\
\hline 422 & 12.531 & 13.468 & 13.321 & 13.364 & -0.937 & 0.147 & -0.043 \\
\hline 424 & 12.173 & 13.194 & 13.326 & 13.408 & -1.021 & -0.132 & -0.082 \\
\hline 425 & 12.857 & 13.733 & 13.846 & 13.983 & -0.876 & -0.113 & -0.137 \\
\hline 426 & 12.572 & 13.549 & 13.702 & 13.826 & -0.977 & -0.153 & -0.124 \\
\hline 427 & 13.090 & 13.932 & 13.570 & 13.587 & -0.842 & 0.362 & -0.017 \\
\hline 428 & 12.996 & 13.819 & 13.967 & 14.215 & -0.823 & -0.148 & -0.248 \\
\hline 430 & 12.969 & 13.947 & 13.991 & 14.215 & -0.978 & -0.044 & -0.224 \\
\hline
\end{tabular}


Table 4. Comparison of AAOmega classifications with those available in the literature.

\begin{tabular}{|c|c|c|c|}
\hline Star & Alias & AAOmega & Published \\
\hline 034 & LH64-6 & B0.7 Ib(n) Nwk & B1 III [M00] \\
\hline 036 & W61 16-6, LH64-7 & $\mathrm{B} 1.5 \mathrm{II}-\mathrm{Ib}(\mathrm{n})$ & B1: III: [M00] \\
\hline 038 & W61 16-7, LH64-33 & B1-1.5 V-III & B1 III [M00] \\
\hline 040 & W61 16-8, LH64-16 & ON2 III(f*) & 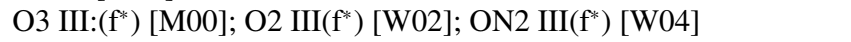 \\
\hline 043 & W61 16-29 & B1 Ib(n) Nwk & B0.2: III: [M00] \\
\hline 047 & SOI 624 & B9 Ib & A1 Iab [SOI] \\
\hline 049 & SOI 625 & A5 II & B9 Ib [SOI] \\
\hline 050 & W61 16-62, LH64-60 & B1 Ib Nwk & B1.5 III [M00] \\
\hline 074 & $\mathrm{Sk}-68^{\circ} 105$ & O9.7 Iab & B0 Ia [M95] \\
\hline 078 & SOI 399 & B3 Iab & $\mathrm{A} 0 \mathrm{Ia}[\mathrm{SOI}]$ \\
\hline 084 & & B1.5 Ib Nwk & B1.5 Ib [M14; their lm0020n19615] \\
\hline 106 & D226-5 & $06.5 \mathrm{~V}((\mathrm{f}))$ & O6.5 V((f)) [O96b] \\
\hline 112 & $\mathrm{Sk}-68^{\circ} 117$ & B1 Iab Nstr & B1 III [M95] \\
\hline 120 & $\mathrm{Sk}-68^{\circ} 119$ & O9-9.2 III-II(n) & O9 V [M95] \\
\hline 142 & BI 214 & O6.5(n)(f)p & O6.5 f [C86]; AAOmega type is from W10 \\
\hline 151 & LH 81-1018 & $09.7 \mathrm{~V}$ & B0.5 III [M00] \\
\hline 155 & BI 220 & O9.7 II(n) & B0.5 III [M95] \\
\hline 159 & W61 28-23 & $\mathrm{O} 3.5 \mathrm{III}\left(\mathrm{f}^{*}\right)$ & O3 V((f)) [M00]; O4 III(f+) [W02]; O3.5 V((f+)) [M05] \\
\hline 161 & BI 217 & $\mathrm{~B} 2.5 \mathrm{Ib}$ & B2 III [M95] \\
\hline 170 & W61 27-1 & B0.2 III(n) & B0.5 III [M00] \\
\hline 179 & ST 3-08 & B0.2 III(n) & B0 III [ST92] \\
\hline 180 & W61 27-7, LH 85-22 & B1 Iab Nwk & B1.5: I [M00] \\
\hline 187 & $\ldots$ & O6n(f)p & AAOmega type is from $\mathrm{W} 10$ \\
\hline 195 & W61 27-31, LH 89-68, ST92 3-62 & B1 Ib Nwk & B1 I [ST92] \\
\hline 200 & Sk $-68^{\circ} 127$ & B0.7 Ib & B0.5 Ia [M95] \\
\hline 214 & $\mathrm{Sk}-69^{\circ} 216 \mathrm{a}$ & O6.5 III(f) & O6 V((f)) [M95] \\
\hline 241 & $\mathrm{Sk}-68^{\circ} 132$ & B0 III(n) & B0 V [M95] \\
\hline 254 & VFTS 016 & O2 III-If* & O2 III-If* [E10] \\
\hline 276 & BI 253, VFTS 072 & $\mathrm{O} 2 \mathrm{~V}-\mathrm{III}(\mathrm{n})\left(\left(\mathrm{f}^{*}\right)\right)$ & $\mathrm{O} 3 \mathrm{~V}$ [M95]; O2 V $\left(\left(\mathrm{f}^{*}\right)\right)$ [W02]; adopted type is from W14 \\
\hline 277 & $\mathrm{Sk}-68^{\circ} 134$ & B1 Ib-Iab(n) Nwk? & B1 Ib [M95] \\
\hline 283 & ST92 1-72, VFTS 171 & O7-8 III((f)) & O8 III [ST92]; O8 II-III(f) [W14] \\
\hline 286 & $\mathrm{Sk}-69^{\circ} 238$ & $\mathrm{O} 7.5 \mathrm{Ib}(\mathrm{f})$ & O6.5 V((f)) [M95] \\
\hline 287 & $\mathrm{Sk}-69^{\circ} 237$ & B1 Ia Nstr & B1 Ia (N str) [F91] \\
\hline 296 & P93-304, VFTS 389 & O9 III(n) & O8 V [M85]; O8 V [WB97]; O9.5 V [W02b]; O9.5 IV [W14] \\
\hline 308 & P93-9017, VFTS 481 & O8.5-9 II & O8.5 V [P93]; O8.5 III [W14] \\
\hline 318 & ST92 5-85 & $\mathrm{O} 8 \mathrm{Ib}(\mathrm{f})$ & O7.5 I(f) [TN98] \\
\hline 320 & ST92 5-82 & $O 7 n(f) p$ & O6.5 III [TN98]; AAOmega type is from W10 \\
\hline 330 & W61 3-9, [M2002] LMC 172231 & O9: V-III + O9.5: V-III & O9.5 III [ST92 5-67, TN98]; O9 V + O9.5 V [M12] \\
\hline 333 & ST92 5-31 & $\mathrm{O} 2-3(\mathrm{n}) \mathrm{f}^{*} \mathrm{p} \mathrm{Nwk}$ & O3 If [TN98]; AAOmega type is from W10 (plus addition of Nwk) \\
\hline 334 & ST92 5-25 & O5-6 V((f))z & O4 V [TN98] \\
\hline 345 & BI 260 & O6.5 II(f)(n) & $\mathrm{O} 7 \mathrm{~V}((\mathrm{f}))[\mathrm{M} 95]$ \\
\hline 352 & F09048 & Mid-O V & O4-6 Vz [F09] \\
\hline 361 & BI 265, F09 082 & $\mathrm{O} 5 \mathrm{III}(\mathrm{fc})$ & O6 V [M95]; O5n(f+)p [F09] \\
\hline 364 & F09088 & $\mathrm{O} 4 \mathrm{III}(\mathrm{f})$ & O4 III(f) [F09] \\
\hline 367 & BI 254 & O5-6 III(f) & O8 V [M95] \\
\hline 368 & $\ldots$ & O7.5n(f)p & AAOmega type is from $\mathrm{W} 10$ \\
\hline 369 & F09 111 & O5-6 Vz & O6: Vz [F09] \\
\hline 374 & $\ldots$ & Early B + Early B & B1 III (SB2) [M14; their lm0031122987] \\
\hline 375 & $\mathrm{Sk}-68^{\circ} 147$ & $\mathrm{~B} 1.5 \mathrm{Ib}$ & B2 II [J01] \\
\hline 376 & Sk-69²68, F09 147 & B1.5 Ia Nwk & BC1.5 Iab [F09] \\
\hline 377 & $\mathrm{Sk}-69^{\circ} 269, \mathrm{~F} 09150$ & $\mathrm{~B} 1.5 \mathrm{Ib}$ & B1 III: [M95]; B1.5 Iab [F09] \\
\hline 380 & $\mathrm{Sk}-69^{\circ} 269 \mathrm{a}$ & $\mathrm{O} 7(\mathrm{n})(\mathrm{f}) \mathrm{p}$ & AAOmega type is from $\mathrm{W} 10$ \\
\hline 381 & F09 152 & O9 III & O9 III [F09] \\
\hline 387 & Sk-69²71, F09 158 & B5 Ia & B4 III-I [F09] \\
\hline 389 & F09 165 & B1 Ib & B1.5-2 III-II [F09] \\
\hline 390 & F09 166 & O9.7 Ia & O9.7 II-Ib [F09] \\
\hline 391 & F09 168 & B0 Ia & O9.7 Iab [F09] \\
\hline 394 & $\mathrm{Sk}-70^{\circ} 111$ & B0.7 Ia & B0.5 Ia [F88,F91] \\
\hline 397 & F09 177 & B1 Ib Nwk & B1.5 Ib [F09] \\
\hline 417 & $\mathrm{Sk}-69^{\circ} 291$ & O9.2 II & B0 III [C86] \\
\hline
\end{tabular}

Notes. Sources of classifications: SOI (Stock et al. 1976); M85 (Melnick 1985); C86 (Conti et al. 1986); F88 (Fitzpatrick 1988); F91 (Fitzpatrick 1991); M95 (Massey et al. 1995); O96b (Oey 1996b); WB97 (Walborn \& Blades 1997); TN98 (Testor \& Niemela 1998 ); M00 (Massey et al. 2000); J01 (Jaxon et al. 2001); W02 (Walborn et al. 2002a); W02b (Walborn et al. 2002b); W04 (Walborn et al. 2004); M05 (Massey et al. 2005); F09 (Fariña et al. 2009); E10 (Evans et al. 2010); W10 (Walborn et al. 2010); M12 (Massey et al. 2012); M14 (Muraveva et al. 2014, which were earlier classifications of the AAOmega spectra by CJE); W14 (Walborn et al. 2014). 